\title{
TU/e EN⿴HONE

\section{Error reduction in two-dimensional pulse-area modulation, with application to computer-generated transparencies}

\section{Citation for published version (APA):}

Bastiaans, M. J., \& Akkermans, A. H. M. (1987). Error reduction in two-dimensional pulse-area modulation, with application to computer-generated transparencies. (EUT report. E, Fac. of Electrical Engineering; Vol. 87-E172). Eindhoven University of Technology.

Document status and date:

Published: 01/01/1987

\section{Document Version:}

Publisher's PDF, also known as Version of Record (includes final page, issue and volume numbers)

\section{Please check the document version of this publication:}

- A submitted manuscript is the version of the article upon submission and before peer-review. There can be important differences between the submitted version and the official published version of record. People interested in the research are advised to contact the author for the final version of the publication, or visit the $\mathrm{DOI}$ to the publisher's website.

- The final author version and the galley proof are versions of the publication after peer review.

- The final published version features the final layout of the paper including the volume, issue and page numbers.

Link to publication

\section{General rights}

Copyright and moral rights for the publications made accessible in the public portal are retained by the authors and/or other copyright owners and it is a condition of accessing publications that users recognise and abide by the legal requirements associated with these rights.

- Users may download and print one copy of any publication from the public portal for the purpose of private study or research.

- You may not further distribute the material or use it for any profit-making activity or commercial gain

- You may freely distribute the URL identifying the publication in the public portal.

If the publication is distributed under the terms of Article 25fa of the Dutch Copyright Act, indicated by the "Taverne" license above, please follow below link for the End User Agreement:

www.tue.nl/taverne

Take down policy

If you believe that this document breaches copyright please contact us at:

openaccess@tue.nl

providing details and we will investigate your claim. 


\section{Eindhoven University of Technology Netherlands}

Error Reduction in

Two-Dimensional Pulse-Area Modulation, with Application to Computer-Generated Transparencies

by

M.J. Bastiaans

and

A.H.M. Akkermans

EUT Report 87-E-172

ISBN 90-6144-172-2

ISSN 0167-9708

May 1987 
Eindhoven University of Technology Research Reports

EINDHOVEN UNIVERSITY OF TECHNOLOGY

Department of Electrical Engineering

Eindhoven The Netherlands

ERROR REDUCTION IN TWO-DIMENSIONAL PULSE-AREA MODULATION, WITH APPLICATION TO COMPUTER-GENERATED TRANSPARENCIES

by

M.J. Bastiaans

and

A.H.M. Akkermans

EUT Report 87-E-172

ISBN 90-6144-172-2

ISSN 0167-9708

Coden: TEUEDE

Eindhoven

May 1987 
CIP-GEGEVENS KONINKLIJKE BIBLIOTHEEK, DEN HAAG

Bastiaans, M.J.

Error reduction in two-dimensional pulse-area modulation, with application to computer-generated transparencies / by M.J. Bastiaans and A.H.M. Akkermans. - Eindhoven: University of Technology. - Fig. (Eindhoven University of Technology research reports / Department of Electrical Engineering, ISSN 0167-9708; 87-E-172)

Met lit. opg., reg.

ISBN 90-6144-172-2

SISO 536.1 UDC $778.38: 681.3$ NUGI 832

Trefw.: computerholografie. 
Abstract

This report deals with the synthesis of band-limited functions that are generated by properly low-pass filtering a regular array of area-modulated unit-height pulses; simply choosing the pulse areas proportional to the corresponding sample values of the band-limited function to be generated, would result in an error. The exact relationship between the pulse areas and the corresponding sample values of the band-limited function to be synthesized, is derived. Error reduction can be achieved by using this relationship to calculate the pulse areas from the required sample values; in principle, a band-limited function can thus be realised to any degree of accuracy. It is shown which amount of error reduction can be obtained, when only a limited number of terms of the exact relationship is taken into account. The application to computer-generated half-tone transparencies is described.

Bastiaans, M.J. and A.H.M. Akkermans

ERROR REDUCTION IN TWO-DIMENSIONAL PULSE-AREA MODULATION, WITH APPLICATION TO COMPUTER-GENERATED TRANSPARENCIES.

Department of Electrical Engineering, Eindhoven University of Technology, Eindhoven, Netherlands, 1987.

EUT-Report $87-\mathrm{E}-172$

This work was performed within the Department of Electrical Engineering, Eindhoven University of Technology, Eindhoven, Netherlands.

Addresses of the authors:

M.J. Bastiaans

Eindhoven University of Technology

Department of Electrical Engineering

P.O. Box 513

5600 MB Eindhoven

Netherlands

A.H.M. Akkermans

Nederlandse Philips Bedrijuen B.V.

Consumer Electronics

P.0. Box 80000

$5600 \mathrm{JA}$ Eindhoven

Netherlands 
Table of contents

Abstract

i ii

1. Introduction 1

2. Generation of a two-dimensional band-1imited function 5

$\begin{array}{ll}3 \text {. The linearized system } & 17\end{array}$

4. The inverse system 27

5. Application to computer-generated transparencies 36

$\begin{array}{ll}\text { References } & 40\end{array}$

$\begin{array}{ll}\text { Tables } & 41\end{array}$ 


\section{Introduction}

The well-known sampling theorem [1] provides a mathematical basis for the synthesis of coherent optical fields by means of computer-generated transparencies $(2-3\}$. The theorem tells us that a band-1imited function can be described completely by its values on a regular array of sample points. Moreover, it shows that a band-limited function whose sample values are known, can be synthesized by a proper low-pass filtering of a regular array of Dirac functions; the Dirac functions must be centered at the sample points and their "masses" must equal the corresponding sample values.

In practice, when synthesizing a band-linited function by low-pass filtering an array of Dirac functions, the practically unrealizable Dirac functions have to be approximated by realizable functions. In the case of computer-generated transparencies, for instance, a Dirac function will often be approximated by a fully transparent dot on an opaque background, the area of the dot being proportional to the mass of the Dirac function. It will be clear that such a dot represents a Dirac function only if the dot size remains sufficiently small. Restricting ourselves to small dot sizes, however, we find ourselves limited in the dynamical range that can be reached in practice. We therefore look for a way that allows us to enlarge the dot size. 
If, in the synthesis procedure mentioned above, we simply substitute for a Dirac function a function that approximates it more or less, an inevitable error will always occur in the band-limited function being generated at the output of the low-pass filter: the function will be "distorted." In a previous paper [4] we have derived a generalized form of the sampling theorem, in which the sample values of the band-limited function to be synthesized are "predistorted"; this generalized sampling theorem should be used in the case that Dirac functions are replaced by practically realizable functions. Applying predistortion, we can, in principle, completely eliminate the distortion that otherwise occurs in the band-limited function. In. the case of computer-generated transparencies, predistortion implies that the dot size (which need not remain small) is no longer proportional to the sample value at the corresponding sample point, but is determined, even in a nonlinear way, by neighbouring sample points, too.

In this report we shall consider the synthesis of coherent optical fields by means of a computer-generated transparency that is based on area-modulation of a regular array of unit-height pulses. In order not to rest too heavily on Ref.4, the derivations given there are partly repeated in this report, but now with emphasis on the special case of pulse-area modulation.

The way in which a two-dimensional band-limited function can 
be synthesized by means of properly low-pass filtering a regular array of area-modulated (circularly-shaped unit-height) pulses is presented in Section 2. In particular, the relationship between the pulse areas and the corresponding sample values is derived [see Eq.(2.18)]. This nonlinear and nonlocal relationship is the main result of Section 2 .

The basic relationship given by Eq. $(2.18)$ is linearized in Section 3. The linearized version provides the basis for a simple predistortion scheme. We investigate which error reduction can be achieved by such a simple predistortion.

If the exror reduction that is obtained by this simple predistortion scheme is not sufficient, a more sophisticated scheme must be derived. We therefore find, in Section 4, the inverse of Eq.(2.18) [see Eq. (4.6), which is the main result of Section 4]. The exror reduction that is achieved by using Eq. (4.6) depends on the number of terms that we take into account. Several cases will be described and it will be shown that a substantial error reduction can be achieved, even if we restrict ourselves to a small number of terms.

The application to computer-generated transparencies is described in Section 5 . In that final section we also describe how we can synthesize, to any degree of accuracy, real or complex light amplitudes by using holographic concepts. 
We conclude this introduction with some renarks about notation. Bold-face lower-case characters will throughout be used to denote (two-dimensional) column vectors whereas bold-face upper-case characters will denote matrices; thus, $\mathbf{x}$ will denote a space vector, $\mathbf{u}$ a spatial-frequency vector, and $\mathbf{j , k}, \mathbf{m}, \mathbf{n}$ integer-valued vectors, while $\mathrm{X}, \mathrm{U}$, and $\mathrm{I}$ denote the sampling matrix, the periodicity matrix, and the unit matrix, respectively [see also Ref.5]. Vector and matrix transposition will be denoted by the superscript ${ }^{t}$; thus $u^{t} x$, with $x=\left[x_{1}, x_{2}\right]^{t}$ and $u=\left[u_{1}, u_{2}\right]^{t}$, denotes the inner product $u_{1} x_{1}+u_{2} x_{2}$. In integrals, the expressions $d x$ and $d u$ are used to denote the products $\mathrm{dx}_{1} \mathrm{dx}_{2}$ and $d u_{1} d u_{2}$, respectively. 


\section{Generation of a two-dimensional band-1imited function}

We consider a two-dimensional function $\varphi(\mathbf{x})$ whose Fourier transform

$\bar{\varphi}(\mathbf{u})=\int \varphi(\mathbf{x}) \exp \left[-\dot{\mathbf{i}} \mathbf{u}^{\mathrm{t}} \mathbf{x}\right] \mathbf{d x}$

has a finite support $\mathbf{\Omega}$, i.e., $\bar{\varphi}(\mathbf{u})$ vanishes outside the frequency interval $u \in \Omega$. Throughout this report, the Fourier transform of a function is denoted by the same symbol as the function itself, but marked by a bar on top of the symbol; furthermore, if not stated otherwise, all integrations and summations extend from $-\infty$ to $+\infty$. The periodic extension of $\dot{\varphi}(\mathbf{u})$, with periodicity matrix $\mathbf{U}$, can be expanded into a Fourier series according to

$\sum_{\mathbf{m}} \bar{\varphi}(\mathbf{u}+\mathbf{U} \mathbf{m})=\operatorname{det}(\mathbf{X}) \sum_{\mathbf{m}} \varphi_{\mathbf{m}} \exp \left[-\mathbf{i} \mathbf{u}^{t} \mathbf{X} \mathbf{m}\right]$,

where a matrix $X$ [and its determinant $\operatorname{det}(X)]$ is introduced, which is related to $U$ through the relation $x^{t} U=2 \pi I$. If the periodicity matrix $\mathbf{U}$ is chosen such that neighbouring replicas of $\bar{\varphi}(\mathbf{u})$ [see Eq. (2.2)] do not overlap, then, for uen, $\bar{\varphi}(\mathrm{u})$ can be expressed in the Fourier expansion given in the right-hand side of Eq. (2.2) 
$\bar{\varphi}(\mathbf{u})=\operatorname{det}(\mathbf{X}) \sum_{\mathbf{m}} \varphi_{\mathbf{n}} \exp \left[-\mathbf{i} \mathbf{u}^{\mathbf{t}} \mathbf{X} \mathbf{m}\right] \quad(\mathbf{u} \in \mathbf{\Omega})$

and the expansion coefficients $\varphi_{\mathbf{m}}$ are given by the relation

$\operatorname{det}(\mathbf{X}) \varphi_{\mathbf{m}}=\frac{1}{\operatorname{det}(\mathbf{U})} \int_{\mathbf{\Omega}} \bar{\varphi}(\mathbf{u}) \exp \left[\mathbf{i} \mathbf{u}^{\mathrm{t}} \mathbf{X} \mathbf{m}\right] \mathbf{d u}$

Since the right-hand side of Eq. (2.4) equals $\operatorname{det}(\mathbf{X}) \varphi(\mathbf{X} \mathbf{m})$, we conclude that the expansion coefficients $\varphi_{m}$ are equal to the sampling values $\varphi\left(\mathbf{X}_{\mathbf{m}}\right)$. Applying an inverse Fourier transformation on Eq.(2.3), we get

$\varphi(\mathbf{x})=\sum_{\mathbf{m}} \varphi_{\mathbf{n}} \frac{1}{\operatorname{det}(\mathbf{U})} \int_{\mathbf{\Omega}} \exp \left[\mathbf{i} \mathbf{u}^{\mathrm{t}}\left(\mathbf{x}-\mathbf{X m}_{\mathbf{m}}\right)\right] \mathbf{d u}$.

Equation (2.5) tells us that $\varphi(\mathbf{x})$ is completely described by its values $\varphi(\mathbf{X m})=\varphi_{\mathbf{m}}$ on the regular array of sampling points $\mathbf{x}=\mathbf{X} \mathbf{m}$, and thus represents the well-known sampling theorem [1] for band-limited functions; hence, the matrix $\mathbf{X}$ can be interpreted as a sampling matrix. If the frequency range $\mathbf{\Omega}$ is such that its periodic extension with periodicity matrix $U$ fills the frequency plane completely (and without overlap, of course), then the area of $\Omega$ is equal to $\operatorname{det}(\boldsymbol{U})$; we shall throughout assume that the condition of complete filling without overlap is met.

It is well known that when the regular array of Dirac 
functions $\sum \varphi_{\mathbf{m}} \delta\left(\mathbf{X}^{-1} \mathbf{x}-\mathbf{m}\right)$ forms the input of a low-pass filter that passes all frequency components in the range $u \in \Omega$ and blocks all other components, the band-limited output signal will have the form of Eq.(2.5). Instead of a sequence of practically unrealizable Dirac functions $\varphi_{\mathbf{m}} \delta\left(\mathbf{X}^{-1} \mathbf{x}-\mathbf{m}\right)$, we usually apply to the input of the low-pass filter an array of practically realizable functions $\mathrm{p}\left(\mathbf{x}^{-1} \mathbf{x}-\mathbf{m} ; \psi_{\mathbf{m}}\right)$, say:

$\psi(\mathbf{x})=\sum_{\mathbf{m}} \mathrm{p}\left(\mathbf{X}^{-1} \mathbf{x}-\mathbf{m} ; \psi_{\mathbf{m}}\right)$.

[In the case of computer-generated transparencies, for instance, we often use an array of area-modulated unit-height pulses.] The function $\mathrm{p}\left(\mathrm{x}^{-1} \mathbf{x} ; \psi\right)$ depends on the variable $\mathbf{x}$, with parameter $\psi$; different values of this parameter determine different members of the set of p-functions, which may differ in their shapes. [In the case of area-modulated unit-height pulses, again, $\psi$ would be a measure of the pulse area.] A p-function may be chosen rather arbitrarily; we only require that its Fourier transform $\bar{p}\left(\mathbf{x}^{t} \mathbf{u} ; \psi\right)$ can be expanded into a Taylor series around the center value $\psi_{\mathrm{c}}$

$$
\overline{\mathrm{p}}\left(\mathbf{x}^{\mathrm{t}} \mathbf{u} ; \psi\right)=\sum_{\mathrm{r}=0}^{\infty} \mathrm{r}_{\overline{\mathrm{p}}}\left(\mathbf{x}^{\mathrm{t}} \mathbf{u}\right)\left(\psi-\psi_{\mathrm{c}}\right)^{\mathrm{r}} .
$$

As an example we consider a function that can be used for 
computer-generated transparencies: a unit-height pulse having a circular shape with variable radius $\left(x^{t} \mathbf{x}\right)^{3 / 2}$

$p\left(x^{-1} x ; \psi\right)= \begin{cases}1 & \text { for } x^{t} x<(1 / \xi \xi)^{2} \psi \\ 0 & \text { for } x^{t} x>(1 / \xi \xi)^{2} \psi\end{cases}$

The constant $\xi$ can be chosen arbitrarily, at least for this moment; we shall relate it to the sampling matrix $X$ in due course. The Fourier transform of this function $p\left(x^{-1} x ; \psi\right)$ reads

$\overline{\mathbf{p}}\left(\mathbf{X}^{t} \mathbf{u} ; \psi\right)=\psi \frac{\pi\left(\xi_{2} \xi\right)^{2}}{\operatorname{det}(\mathbf{X})} \frac{J_{1}\left(\xi_{2} \sigma \xi \sqrt{\psi}\right)}{3_{4} \sigma \xi \sqrt{\psi}}$

with $\sigma=\left(\mathbf{u}^{t} \mathbf{u}\right)^{\frac{1}{2}}$, and can be expanded in the form of Eq. (2.7) with

${ }_{\vec{p}}\left(\mathbf{X}^{t} \mathbf{u}\right)=\frac{\pi\left(3_{\xi} \xi\right)^{2}}{\operatorname{det}(\mathbf{X})} \psi_{\mathrm{c}} \frac{\mathrm{J}_{1}\left(\xi_{\xi} \sigma \xi \sqrt{\psi_{c}}\right)}{3_{\sigma} \sigma \xi \sqrt{\psi_{c}}}$

$\mathrm{I}_{\mathrm{p}}\left(\mathbf{x}^{\mathrm{t}} \mathbf{u}\right)=\frac{\pi\left(w_{\xi} \xi\right)^{2}}{\operatorname{det}(\mathbf{X})} \mathrm{J}_{0}\left(1_{\xi} \sigma \xi \sqrt{\psi_{\mathrm{c}}}\right)$

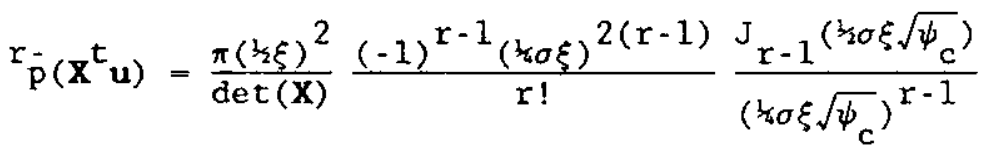

$(x=2,3, \ldots)$

Eqs. (2.10a,b) are, in fact, special cases of Eq.(2.10c). Note that in the special case of $\psi_{c}: 0$, Eqs. (2.10) reduce to 
${ }_{p}^{0}\left(x^{t} \mathbf{u}\right)-0$

$1_{p}\left(\mathbf{x}^{t} \mathbf{u}\right)=\frac{\pi\left(3_{\xi} \xi\right)^{2}}{\operatorname{det}(\mathbf{X})}$

$r_{p}\left(X^{t} u\right)=\frac{\pi\left(3_{2} \xi\right)^{2}}{\operatorname{det}(X)} \frac{(-1)^{r-1}\left(\frac{1}{\pi} \sigma \xi\right)^{2(r-1)}}{r !(r-1) !} \quad(r=2,3, \ldots)$.

We now use the sequence of p-functions given by Eq. (2.6), as the input signal of a low-pass filter that passes all frequency components in the interval $u \in \Omega$ and blocks all frequency components outside that interval. The band-limited output signal of the low-pass filter can be represented in the form of Eq.(2.5). In this section we shall derive the relationship between the sample values $\varphi_{\mathbf{m}}$ of the output signal $\varphi(\mathbf{x})$ and the parameter values $\psi_{\mathrm{m}}$ of the input signal $\psi(\mathrm{x})$. We shall find this relationship via the frequency domain.

The Fourier transform of the input signal $\psi(x)$ given by Eq. (2.6), reads

$$
\begin{aligned}
\bar{\psi}(\mathbf{u}) & =\operatorname{det}(\mathbf{X}) \sum_{\mathbf{m}} \dot{\mathrm{p}}\left(\mathbf{X}^{\mathrm{t}} \mathbf{u} ; \psi_{\mathbf{m}}\right) \exp \left[-\mathbf{i} \mathbf{u}^{\mathrm{t}} \mathbf{X}_{\mathbf{m}}\right]- \\
& =\operatorname{det}(\mathbf{X}) \sum_{\mathbf{m}} \exp \left[-\mathbf{i} \mathbf{u}^{\mathrm{t}} \mathbf{X} \mathbf{n}\right] \sum_{\mathbf{r}=0}^{\infty} r_{\bar{p}}\left(\mathbf{X}^{\mathrm{t}} \mathbf{u}\right)\left(\psi_{\mathbf{m}}-\psi_{\mathbf{c}}\right)^{\mathbf{r}},
\end{aligned}
$$


where the Taylor series expansion [Eq.(2.7)] has been substituted. The Fourier transform of the band-limited output signal $\varphi(x)$ is given by Eq.(2.3). We require that in the frequency interval $u \in \mathbb{\Omega}$ these two Fourier transforms [Eqs.(2.3) and (2.12)] are identical. We now expand ${ }_{\bar{p}}\left(x^{t} u\right)$ in a Fourier series in this interval, yielding

$r_{\dot{p}}\left(x^{t} u\right)=\sum_{k}^{r} p_{k} \exp \left[-i u^{t} \mathbf{x k}\right] \quad(u \in \boldsymbol{\Omega})$

with expansion coefficients ${ }^{r} p_{\mathbf{k}}$ given by the relation

$r_{p_{k}}=\frac{1}{\operatorname{det}(\mathbf{D})} \int_{\mathbf{\Omega}} r_{\dot{p}}\left(\mathbf{x}^{t} \mathbf{u}\right) \exp \left[i \mathbf{u}^{t} \mathbf{x k}\right] \mathbf{d u} ;$

note the small difference between Eqs. (2.3) and (2.4) on the one hand and Eqs.(2.13) and (2.14) on the other. Substituting Eq.(2.13) into Eq.(2.12), we arrive at

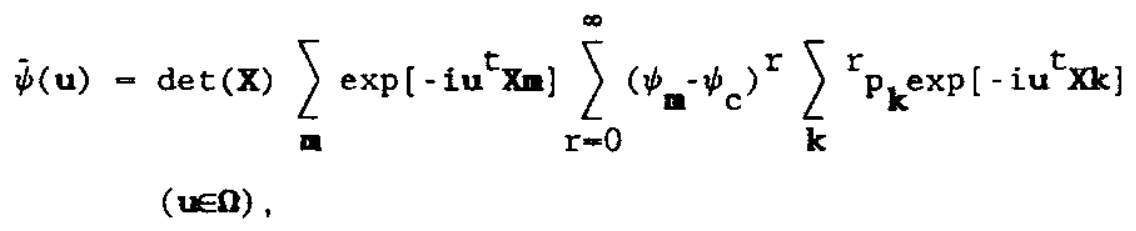

which, after a suitable transformation of the sumnation variables (viz., first making the substitutions $\mathbf{m}$ and $\mathbf{k} \rightarrow \mathbf{m}-\mathbf{k}$, and then the substitutions $\mathbf{n} \rightarrow$ and $\mathbf{k} \rightarrow \mathbf{k}$ ), can be expressed as 
$\bar{\psi}(\mathbf{u})=\operatorname{det}(\mathbf{X}) \sum_{\mathbf{m}} \exp \left[-\mathbf{i} \mathbf{u}^{\mathrm{t}} \mathbf{X} \mathbf{m}\right] \sum_{\mathbf{r}=0}^{\infty} \sum_{\mathbf{k}} \mathrm{r}_{\mathbf{m}-\mathbf{k}}\left(\psi_{\mathbf{k}}-\psi_{\mathbf{c}}\right)^{\mathrm{r}} \quad(\mathbf{u} \in \mathbf{\Omega})$.

Identity of the Fourier transform [Eq.(2.3)] of the output signal and the Fourier transform [Eq.(2.16)] of the input signal in the interval $\mathbf{u} \in \mathbf{\Omega}$, implies the important relationship

$\varphi_{\mathbf{m}}-\sum_{r=0}^{\infty} \sum_{\mathbf{k}} \mathrm{r}_{\mathbf{m}-\mathbf{k}}\left(\psi_{\mathbf{k}}-\psi_{\mathrm{c}}\right)^{\mathrm{r}}-{ }^{0} \bar{p}(\mathbf{0})+\sum_{\mathrm{r}=1}^{\infty} \sum_{\mathbf{k}} \mathrm{r}_{\mathrm{m}-\mathbf{k}}\left(\psi_{\mathbf{k}}-\psi_{\mathrm{c}}\right)^{\mathrm{r}}$

between the output sample values $\varphi_{m}$ and the input parameter values $\psi_{\mathbf{m}}$. With $\varphi_{\mathrm{c}}={ }^{0} \overline{\mathrm{p}}(\mathbf{0})$, Eq. (2.17) can be expressed in the final form

$\varphi_{\mathbf{m}}-\varphi_{\mathrm{c}}=\sum_{\mathrm{r}=1}^{\infty} \sum_{\mathbf{k}} \mathrm{r}_{\mathbf{m}-\mathbf{k}}\left(\psi_{\mathbf{k}}-\psi_{\mathrm{c}}\right)^{\mathrm{r}}$

Let us now consider again the case of area-modulated circularly-shaped unit-height pulses described by Eqs. (2.8)-(2.11). The offset value $\varphi_{c}=\bar{p}(0)$ follows directly from Eq. (2.10a) and takes the value $\varphi_{c}=\left[\pi\left(\frac{1}{2} \xi\right)^{2} / \operatorname{det}(X)\right] \psi_{c}$. The coefficients ${ }^{r} p_{k}(r-1,2, \ldots)$ follow from applying the operation described by Eq. (2.14) to the functions $r_{p}\left(x^{t} u\right)$ defined by Eqs. $(2.10 b, c)$. In order to calculate the coefficients ${ }^{r} p_{k}$ we must choose the frequency interval $\mathbf{\Omega}$ and the periodicity matrix $\mathbf{U}$ (and 
sampling matrix $\mathrm{X}$ ). We shall throughout consider two cases:

(i) rectangular sampling, with the sampling matrix $\mathrm{X}$ and the periodicity matrix $\mathbf{U}$ equal to

$\mathbf{X}=\left[\begin{array}{ll}\xi & 0 \\ 0 & \xi\end{array}\right] \quad \mathbf{U}=\left[\begin{array}{cc}2 \pi / \xi & 0 \\ 0 & 2 \pi / \xi\end{array}\right]$

respectively, and the frequency interval $\mathbf{\Omega}$ as depicted in Fig.1, and

(ii) hexagonal sampling, with the sampling matrix $x$ and the periodicity matrix $\mathbf{U}$ equal to

$\mathbf{X}=\left[\begin{array}{cc}\xi / 2 & \xi / 2 \\ \xi \sqrt{3} / 2 & -\xi \sqrt{3} / 2\end{array}\right] \quad \mathbf{U}=\left[\begin{array}{cc}2 \pi / \xi & 2 \pi / \xi \\ 2 \pi / \xi \sqrt{3} & -2 \pi / \xi \sqrt{3}\end{array}\right]$

respectively, and the frequency interval $\mathbf{n}$ as depicted in Fig.2 [see also Ref.5]. Note that the sampling matrix has been chosen in such a way that a periodic extension of a circle with radius $\frac{1}{2 \xi}$ [and normalized area $\psi=1$, see Eq.(2.8)] yields an array of circles that touch but do not overlap each other (see Fig. 3 for the rectangular case and Fig. 4 for the hexagonal case). Since $\pi\left(\frac{1}{2} \xi\right)^{2}$ represents the area of such a circle and $\operatorname{det}(\mathrm{X})$ represents the area of an elementary cell of the periodic array, the quantity $\pi\left(\frac{1}{2} \xi\right)^{2} / \operatorname{det}(\mathbf{X})$ has a clear physical meaning: it expresses the packing density when a plane is packed with circles that touch but do not overlap; in the remainder of the report we shall 


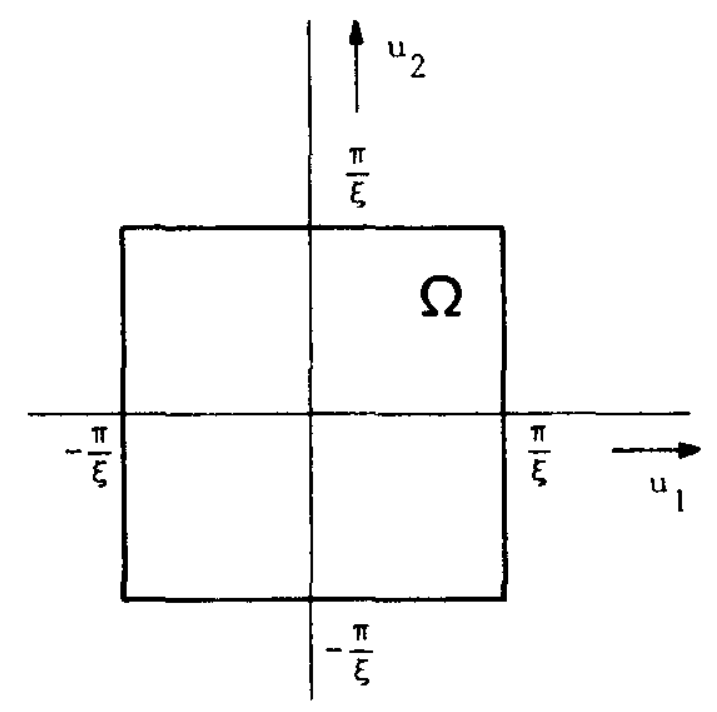

Figure 1 . The support $\Omega$ for rectangular sampling.

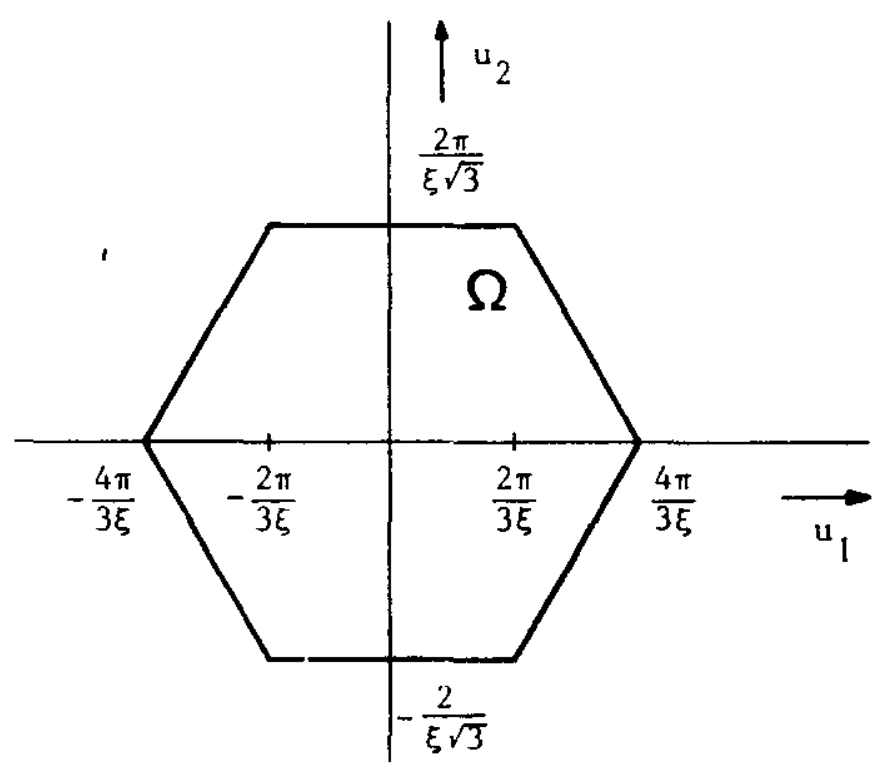

Figure 2. The support $\Omega$ for hexagonal sampling. 


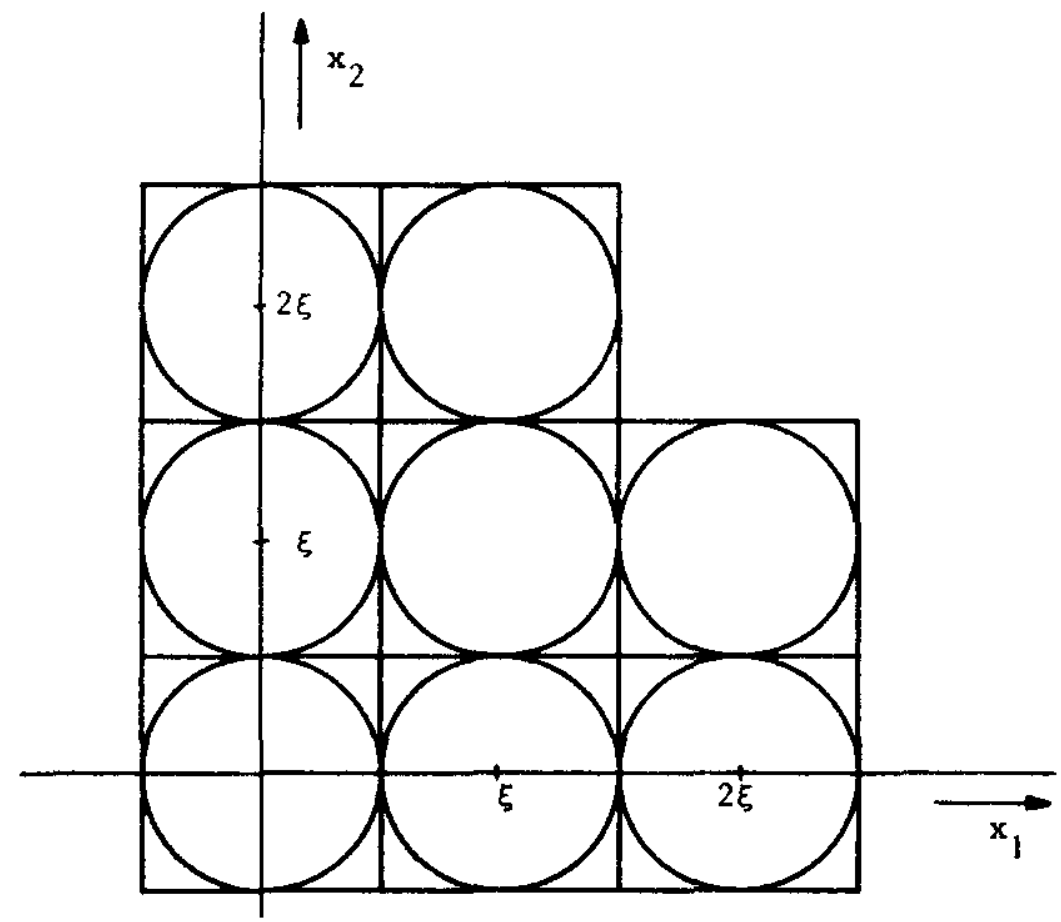

Figure 3. The sampling geometry for rectangular sampling.

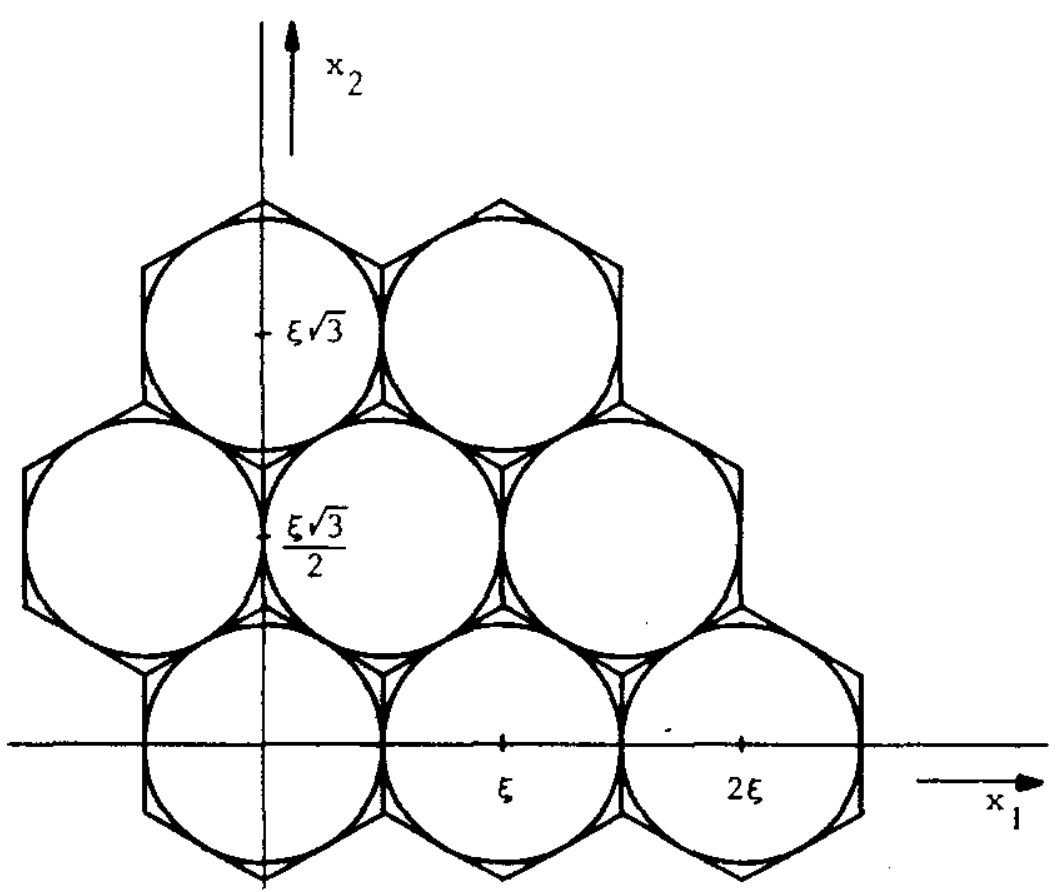

Figure 4. The sampling geometry for hexagonal sampling. 
throughout denote this quantity by $D$. Note that for rectangular sampling $D$ equals $D_{\text {rect }}=\pi / 4=0.78540$, while for hexagonal sampling $D$ equals $D_{\text {hex }}=\pi / 2 \sqrt{3}=0.90690$; this shows an advantage of hexagonal sampling over rectangular sampling, viz., a higher packing density.

We have calculated the coefficients ${ }^{r} p_{k}$ for the special case of $\psi_{c}=0$. Note that for this special value of $\psi_{c}$ the first-order term ${ }^{1-}\left(\mathbf{x}^{\mathrm{t}} \mathbf{u}\right)=\pi\left(\frac{1}{2} \xi\right)^{2} / \operatorname{det}(\mathbf{X})=\mathrm{D}$ is independent of the frequency $\mathbf{u}$ [see Eq.(2.11b)] and hence the first-order coefficients ${ }_{p_{k}}$ vanish for all values of $\mathbf{k}$ except for $\mathbf{k}=0$; the coefficient ${ }^{1} \mathrm{P}_{0}$ takes the value $D$, which equals $D_{\text {rect }}=\pi / 4=0.78540$ for rectangular sampling and $D_{\text {hex }}=\pi / 2 \sqrt{3}=0.90690$ for hexagonal sampling. The higher-order coefficients ${ }^{r} P_{k}(r-2,3)$ for small values of $\mathbf{k}=\left[k_{1}, k_{2}\right]^{t}$ are listed in Tables $1 \mathrm{a}$ and $1 \mathrm{~b}$ for the rectangular case (in which case the coefficients possess 8-fold symmetry, with symmetry axes $k_{1}=0, k_{2}=0, k_{1}-k_{2}$ and $k_{1}--k_{2}$ ) and in Tables 2a and $2 b$ for the hexagonal case (in which case the coefficients possess 12 -fold symmetry, with symmetry axes $k_{1}=0, k_{2}=0, k_{1}=k_{2}$, $k_{1}=-k_{2}, k_{1}=2 k_{2}$ and $k_{2}=2 k_{1}$ ). In principle, we can find analytic expressions for ${ }^{r} \mathrm{p}_{\mathbf{k}}$, which we did for the second-order coefficients ${ }^{2} \mathrm{p}_{\mathbf{k}}$. For rectangular sampling we have (with $0 \leq \mathrm{k}_{1} \leq \mathrm{k}_{2}$; the remaining coefficients follow from the symmetry properties) 


$$
\begin{array}{ll}
{ }^{2} \mathrm{p}_{\mathbf{k}}=-\frac{\pi^{3}}{192} & \text { for } \mathrm{k}-[0,0]^{\mathrm{t}} \\
{ }^{2} \mathrm{p}_{\mathbf{k}}=-\frac{\pi(-1)^{\mathrm{m}}}{64 \mathrm{~m}^{2}} & \text { for } \mathrm{k}-[0, \mathrm{~m}]^{\mathrm{t}} \text { with } \mathrm{m}-1,2, \ldots \\
{ }^{2} \mathrm{p}_{\mathbf{k}}=0 & \text { for } \mathrm{k}-[\mathrm{m}, \mathrm{n}]^{\mathrm{t}} \text { with } \mathrm{n}-1,2, \ldots \text { and } 0<\mathrm{m} \leq \mathrm{n} . \quad(21 \mathrm{a})
\end{array}
$$

For hexagonal sampling we have (with $0 \leq 2 \mathrm{k}_{1} \leq \mathrm{k}_{2}$; the remaining coefficients follow from the symmetry properties

$$
\begin{aligned}
& { }^{2} p_{k}=-\frac{5 \pi^{3}}{432 \sqrt{3}} \quad \text { for } k=[0,0]^{t} \\
& { }^{2} p_{k}=-\frac{\pi}{144 \sqrt{3} m^{2}} \quad \text { for } k=[m, 2 m]^{t} \text { with } m=1,2, \ldots \\
& { }^{2} p_{k}=\frac{1}{96(m-n)^{2}}[4 f(m+n)+f(2 m-n)+f(2 n-m)]
\end{aligned}
$$

$$
\text { for } k=[m, n]^{t} \text { with } n-1,2, \ldots \text { and } 0 \leq 2 m<n,(2.22 c)
$$

where we have introduced the function $f(m)=2 \sin (2 \pi m / 3) / m \sqrt{3}$.

Equation (2.18) explicitly expresses the sample values $\varphi_{\mathbf{m}}$ of the output signal in terms of the parameter values $\psi_{m}$ of the input signal, and can be interpreted as the Volterra series [6-7] describing the input-output relationship of a nonlinear system. In the next section we will study which errors occur when we approximate this nonlinear system by the linear term of its Volterra series. 


\section{The linearized system}

In this section we investigate which errors occur when the nonlinear system equation $(2.18)$ is approximated by its linear term

$\gamma_{\mathbf{m}}-\varphi_{c}=\sum_{\mathbf{k}}{ }_{p_{m-k}}\left(\psi_{k}-\psi_{c}\right)$

The error between the output signal $\gamma_{m}$ of the linearized system [described by Eq.(3.1)] and the output signal $\varphi_{\mathbf{m}}$ of the exact system [described by Eq.(2.18)], is given by the higher-order terms of Eq.(2.18)

$\varphi_{\mathbf{m}}-\gamma_{\mathbf{m}}=\sum_{\mathrm{r}=2}^{\infty} \sum_{\mathbf{k}} \mathrm{r}_{\mathbf{m}-\mathbf{k}}\left(\psi_{\mathbf{k}}-\psi_{\mathrm{c}}\right)^{\mathrm{r}}$.

We will study this error for the case of area-modulated circular unit-height pulses. Since in that case $\varphi_{c}={ }^{0} \bar{p}(0)=D \psi_{c}$ and ${ }^{1} \bar{p}(0)=D$ [see Eqs. $(2.10 a, b)]$, we can as well express the linearized system in the form

$\gamma_{\mathbf{m}}=\sum_{\mathbf{k}}^{1} \mathrm{p}_{\mathbf{m}-\mathbf{k}} \mathbf{k}_{\mathbf{k}}$

the coefficients ${ }^{1} p_{k}$ still depend, of course, upon the choice of the center value $\psi_{\mathrm{c}}$. 
Let us first consider the case $\psi_{c}-0$, for which case the coefficients ${ }^{r} p_{k} \quad(r-2,3)$ are listed in Tables $1 a, b$ for rectangular sampling and in Tables $2 a, b$ for hexagonal sampling; we note that for this value of $\psi_{c}$ the linearized system reduces to $\gamma_{m}=D \psi_{m}$. An absolute upper bound for the error $\varphi_{m}{ }^{-\gamma_{m}}$ is now given by the expression

$$
\left|\varphi_{\mathbf{m}}-\gamma_{\mathbf{m}}\right| \leq \sum_{\mathrm{r}=2}^{\infty}\left|\sum_{\mathbf{k}}{ }^{\mathrm{r}} \mathrm{p}_{\mathbf{m}-\mathbf{k}}{ }_{\mathbf{k}}{ }^{\mathrm{r}}\right|
$$

where we remark that the parameter $\psi$ is restricted to the interval $0 \leq \psi \leq 1$, since in the case of area-modulated circular unit-height pulses we do not want to have pulses with negative areas nor pulses that overlap. Each of the summations $\sum{ }_{p} p_{m-k}{ }_{k}{ }^{r}$ $(r=2,3, \ldots)$ that arise in the right-hand side of Eq. (3.4), takes its maximum absolute value for $=0$, if $\psi_{k}$ is chosen equal to 1 for those values of $k$ for which ${ }^{\mathrm{r}_{p_{k}}}$ is positive and equal to 0 for those values of $\mathbf{k}$ for which ${ }^{\mathrm{r}} \mathrm{p}_{\mathbf{k}}$ is negative. The second-order term $\sum{ }^{2} \mathrm{p}_{\mathbf{m}-\mathbf{k}} \psi_{\mathbf{k}}{ }^{2}$ thus yields an error of 0.24223 in the rectangular case and 0.43071 in the hexagonal case; since the higher-order terms are much smaller, the absolute upper bound for the right-hand side of Eq. (3.4) has the same order of magnitude. of course, such a large error value will not arise in practice. However, straightforward calculation of the propagation of a rectangular array of pulses with area values that alternate between a high value $\psi_{\mathrm{h}}$ and a low value $\psi_{1}$ according to 
$\psi_{\mathrm{m}}=\frac{\psi_{\mathrm{h}}+\psi_{1}}{2}+\frac{\psi_{\mathrm{h}}{ }^{-\psi_{1}} 1}{2} \cos \left[\pi\left(\mathrm{m}_{1}+\mathrm{m}_{2}\right)\right]$

yields the rectangular array of sample values

$$
\begin{aligned}
& \varphi_{\mathbf{m}}=\sum_{\mathrm{r}=1}^{\infty}\left(\mathrm{r}_{\bar{p}(0,0)} \frac{\psi_{\mathrm{h}}^{\mathrm{r}+\psi_{1}^{\mathrm{r}}}}{2}+\mathrm{r}_{\overline{\mathrm{p}}(\pi, \pi)} \frac{\psi_{\mathrm{h}}^{\mathrm{r}-\psi_{1}^{\mathrm{r}}}}{2} \cos \left[\pi\left(\mathrm{m}_{1}+\mathrm{m}_{2}\right)\right]\right)= \\
& =\frac{\pi}{4}\left(\frac{\psi_{h}+\psi_{1}}{2}+\frac{\sqrt{2 \psi_{h}} J_{1}\left(\pi \sqrt{\psi_{h} / 2}\right)-\sqrt{2 \psi_{1}} J_{1}\left(\pi \sqrt{\psi_{1} / 2}\right)}{\pi} \cos \left[\pi\left(m_{1}+m_{2}\right)\right]\right),
\end{aligned}
$$

whereas the hexagronal input array

$\psi_{\mathbf{m}}=\frac{\psi_{\mathrm{h}}+2 \psi_{1}}{3}+\frac{\psi_{\mathrm{h}}-\psi_{1}}{3} 2 \cos \left[\frac{2 \pi}{3}\left(\mathrm{~m}_{1}+\mathrm{m}_{2}\right)\right]$

yields the hexagonal output array

$$
\begin{aligned}
& \varphi_{\mathbf{m}}=\frac{\pi}{2 \sqrt{3}}\left[\frac{\psi_{\mathrm{h}}+2 \psi_{1}}{3}+\frac{\sqrt{\psi_{\mathrm{h}}} \mathrm{J}_{1}\left(2 \pi \sqrt{\psi_{\mathrm{h}}} / 3\right)-\sqrt{\psi_{1}} \mathrm{~J}_{1}\left(2 \pi \sqrt{\psi_{1}} / 3\right)}{\pi}\right. \\
& \left.2 \cos \left[\frac{2 \pi}{3}\left(m_{1}+m_{2}\right)\right]\right) .
\end{aligned}
$$

Comparing these output signals [Eqs.(3.6)] with the signals that arise at the output of the linearized system, shows that in the practical case $\psi_{\mathrm{h}}=1$ and $\psi_{1}=0$ the error $\gamma_{0}-\varphi_{0}$ takes the value 
$\pi / 8-\mathrm{J}{ }_{1}(\pi / \sqrt{2}) / 2 \sqrt{2}=0.19740$ for rectangular sampling and the value $\pi / 3 \sqrt{3}-J_{1}(2 \pi / 3) / \sqrt{3}=0.27633$ for hexagonal sampling. We remark that the arrays given by Eqs. (3.5) and (3.6) have Fourier transforms with components at the origin and at the vertices of the intervals $\Omega$, i.e., with $\sigma=\left(u^{t} u\right)^{\frac{1}{2}}=\pi \sqrt{2} / \xi$ for rectangular sampling and with $\sigma=\left(u^{t} u\right)^{3 / 2}=4 \pi / 3 \xi$ for hexagonal sampling (see Figs.1 and 2). We conclude that the simple approximation $\gamma_{m}=D \psi_{m}$ might yield large errors.

The reason for the large errors that arise in the case of $\psi_{c}=0$ is, of course, the fact that $\psi$ may take values in the interval $0 \leq \psi \leq 1$, whereas we have expanded $\overline{\mathrm{p}}\left(\mathrm{X}^{\mathrm{t}} \mathrm{u} ; \psi\right)$ in a Taylor series around the center value $\psi_{\mathrm{c}}=0$. We might expect that an expansion around a center value somewhere in the middle of the interval would yield a much better result. We now try to find an optimum for this center value.

Let us investigate how close the linearized system given by Eq. (3.3) with $\psi_{\mathrm{c}} \neq 0$, resembles the exact system given by Eq. (2.18). We therefore apply to the linearized system the array of alternating parameter values given by Eqs.(3.5), for which the exact system yields the array of alternating sample values given by Eqs.(3.6). The linearized system responds to this input array with the output array

$\gamma_{\mathrm{m}}=\frac{\pi}{4}\left(\frac{\psi_{\mathrm{h}}+\psi_{1}}{2}+\mathrm{J}_{0}\left(\pi \sqrt{\psi_{\mathrm{c}} / 2}\right) \frac{\psi_{\mathrm{h}}{ }^{-\psi_{1}}}{2} \cos \left[\pi\left(\mathrm{m}_{1}+\mathrm{m}_{2}\right)\right]\right)$ 
in the rectangular case and with the output array

$\gamma_{\mathbf{m}}=\frac{\pi}{2 \sqrt{3}}\left[\frac{\psi_{\mathrm{h}}+2 \psi_{1}}{3}+\mathrm{J}_{0}\left(2 \pi \sqrt{\psi_{\mathrm{c}}} / 3\right) \frac{\psi_{\mathrm{h}}-\psi_{1}}{3} 2 \cos \left[\frac{2 \pi}{3}\left(\mathrm{~m}_{1}+\mathrm{m}_{2}\right)\right]\right)$

in the hexagonal case. Comparing Eqs.(3.6) and (3.7) shows the error $\varphi_{m}-\gamma_{m}$ between the two output sequences. When we substitute the values of $\psi_{\mathrm{h}}$ and $\psi_{1}$ for which this error takes its maximum absolute value, and then minimize this maximum error with respect to $\psi_{c}$, we get an optimum for $\psi_{c}$. We thus find for rectangular sampling the optimum value when $\psi_{c}$ satisfies the relation

$J_{0}\left(\pi \sqrt{\psi_{c} / 2}\right)-2 \frac{J_{1}(\pi / \sqrt{2})}{\pi / \sqrt{2}}$,

i.e., for $\psi_{c}=0.47183$, for which value the error becomes 0.04419 when either $\psi_{\mathrm{h}}$ or $\psi_{1}$ equals $\psi_{\mathrm{c}}$ while the other one equals either 1 or 0 ; for hexagonal sampling we find the optimum value when $\psi_{c}$ satisfies the relation

$J_{0}\left(2 \pi \sqrt{\psi_{c}} / 3\right)=2 \frac{J_{1}(2 \pi / 3)}{2 \pi / 3}$

i.e. for $\psi_{\mathrm{c}}=0.47522$, for which value the error becomes 0.06267 when either $\psi_{\mathrm{h}}$ or $\psi_{1}$ equals $\psi_{\mathrm{c}}$ while the other one equals either 1 or 0 . When we compare these error values with the errors that we found for $\psi_{\mathrm{c}}=0$, we conclude that the linearized system with the optimum choice of $\psi_{c}$ according to Eqs.(3.8), is better than 
the simple system $\boldsymbol{\gamma}_{\mathbf{m}}=\mathrm{D} \psi_{\mathrm{m}}$ by a factor of about 4.4 . The coefficients ${ }^{1} p_{k}$ for these optimum values of $\psi_{c}$ are listed in Table $3 a$ for rectangular sampling and in Table $4 a$ for hexagonal sampling.

The concept of the linearized system allows us to achieve an error reduction when we want to generate a band-limited function by low-pass filtering a regular array of area-modulated unit-height pulses. Let the sample values of the band-1imited function to be generated, be denoted by $\gamma_{\mathbf{m}}$. If we simply take the widths $\psi_{\mathbf{m}}$ of the pulses equal to $\gamma_{\mathbf{m}} / D$, large errors may occur, as we have shown in the first part of this section. These errors can be reduced when we first apply a linear predistortion to the sample values:

$\psi_{\mathbf{m}}=\sum_{\mathbf{k}}^{1} \mathrm{q}_{\mathbf{m}-\mathbf{k}} \boldsymbol{\gamma}_{\mathbf{k}}$

where ${ }^{1} q_{k}$ is the inverse operator of ${ }^{1} p_{k}$ as described in Eq. (3.3). As we have shown, an error reduction by a factor of 4.4 can thus be achieved. The coefficients ${ }^{1} q_{k}$ are 1 isted in Table $3 b$ for rectangular sampling and Table $4 \mathrm{~b}$ for hexagonal sampling.

Until now, we have based the analysis on the Taylor series expansion given by Eq.(2.7), and we have found an optimum linearized system by finding the optimum value of $\psi_{c}$. If the 
parameter values $\psi_{m}$ are restricted to a certain interval, there exists a different way to find a linearized system, viz., via powex series economization [8] of Eq.(2.7) using an expansion into (properly shifted and scaled) Tschebyscheff polynomials. To show this, let us confine ourselves to the parameter value interval $0 \leq \psi \leq 1$, in which case we can express $\overline{\mathrm{p}}\left(\mathrm{x}^{\mathrm{t}} \mathbf{u} ; \psi\right)$ in the form of a series of the Tschebyscheff polynomials $T_{r}^{*}(\psi)$ [c.f. Eq. (2.7)] :

$\overline{\mathrm{p}}\left(\mathbf{x}^{\mathrm{t}} \mathbf{u} ; \psi\right)=\sum_{\mathrm{r}=0}^{\infty} \mathrm{x}_{\overline{\mathrm{h}}}\left(\mathrm{x}^{\mathrm{t}} \mathbf{u}\right) \mathrm{T}_{\mathrm{r}}^{*}(\psi)$.

Redoing the analysis of section 2 now results in the system representation [c.f. Eq.(2.18)]

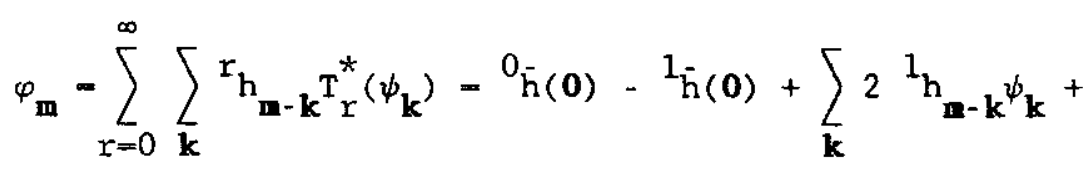

$$
\begin{aligned}
& +\sum_{r=2}^{\infty} \sum_{\mathbf{k}}^{r_{h}}{ }_{m-k^{T}} T_{r}^{*}\left(\psi_{k}\right)
\end{aligned}
$$

where use has been made of the properties of Tschebyscheff polynomials $\mathrm{T}_{0}^{*}(\psi)=1$ and $\mathrm{T}_{1}^{*}(\psi)=2 \psi-1$; the coefficients $\mathrm{r}_{\mathrm{h}_{\mathrm{k}}}$ are the Fourier series expansion coefficients of ${ }^{r} \bar{h}\left(x^{t} \mathbf{u}\right)$ [c.f. Eq. (2.14)]. The first and second terms in the right-hand side of 
Eq. (3.11) represent a mere offset for the output value; the third term represents the linearized system that we are looking for, whereas the last term represents the error between the exact system and the linearized system. We shall write the linearized system that we have found via the Tschebyscheff polynomials in the form

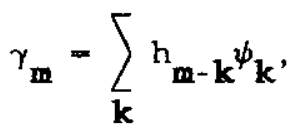

where $h_{k}=2 h_{k}$.

As an example we consider again the case of area-modulated circular unit-height pulses, described by Eqs.(2.8)-(2.11). In that case we have [using Eq.6.681.1 in Ref.9]

$$
\begin{aligned}
& \mathrm{O}_{\overline{\mathrm{h}}}\left(\mathbf{X}^{t} \mathbf{u}\right)=\frac{1}{\pi} \int_{0}^{1} \overline{\mathrm{p}}\left(\mathbf{X}^{t} \mathbf{u} ; \psi\right) \mathrm{T}_{0}^{*}(\psi)\left(\psi-\psi^{2}\right)^{-\frac{2 \xi}{\mathrm{d}} \mathrm{d} \psi-} \\
& =\frac{\pi \xi}{\sigma \operatorname{det}(\mathrm{X})} \mathrm{J}_{1}(\xi \sigma \xi) \mathrm{J}_{0}\left(\frac{1}{\sigma} \sigma \xi\right) \\
& I_{\bar{h}}\left(x^{t} \mathbf{u}\right)=\frac{2}{\pi} \int_{0}^{1} \dot{p}\left(x^{t} \mathbf{u} ; \psi\right) \mathrm{T}_{1}^{*}(\psi)\left(\psi-\psi^{2}\right)^{-\frac{1}{2}} \mathrm{~d} \psi= \\
& =\frac{\pi \xi}{\sigma \operatorname{det}(\mathrm{X})} \mathrm{J}_{1}\left(\frac{3}{\sigma} \sigma \xi\right)\left[\mathrm{J}_{0}(\xi \sigma \xi)-\mathrm{J}_{2}\left(\frac{3}{4} \sigma \xi\right)\right] ;
\end{aligned}
$$


hence ${ }^{0} \bar{h}(0)={ }^{1} \bar{h}(0)=0$ and

$\bar{h}\left(x^{t} \mathbf{u}\right)=2^{1} \bar{h}\left(x^{t} \mathbf{u}\right)=$

$$
=\frac{\pi\left(\frac{1}{2} \xi\right)^{2}}{\operatorname{det}(\mathrm{X})} \frac{2 \mathrm{~J}_{1}\left(\frac{3}{4} \sigma \xi\right)}{3_{4} \sigma \xi}\left[2 \mathrm{~J}_{0}(\xi \sigma \xi)-\frac{2 \mathrm{~J}_{1}^{\left(\frac{1}{4} \sigma \xi \xi\right)}}{3_{4} \sigma \xi}\right] .
$$

The coefficients $h_{\mathbf{k}}$ of the linearized system follow by expanding $\tilde{h}\left(\mathbf{X}^{t} \mathbf{u}\right)$ into a Fourier series [see Eq. (2.14)]; these coefficients are listed in Table 5a for rectangular sampling and in Table 6a for hexagonal sampling. Note the close resemblance between the coefficients $h_{k}$ and the coefficients ${ }^{1} p_{k}$ that we found in the case of the optimum linearized system [see Tables $3 a$ and 4a]. The inverse of the linearized system described by Eq.(3.12) can be expressed as

$\psi_{\mathbf{m}}=\sum_{\mathbf{k}} \mathrm{g}_{\mathbf{m}-\mathbf{k}} \mathbf{\gamma}$,

where $g_{\mathbf{k}}$ is the inverse operator of $h_{k}$; the coefficients $g_{k}$ are listed in Table $5 \mathrm{~b}$ for rectangular sampling and in Table $6 \mathrm{~b}$ for hexagonal sampling.

To investigate how close, in the case of area-modulated circular unit-height pulses, the latter version of the linearized system resembles the exact system, we apply to this system again the alternating input array given by Eqs.(3.5). The linearized 
system responds with the output array given by Eqs.(3.7), but now with $\mathrm{J}_{0}\left(\pi \sqrt{\psi_{\mathrm{c}} / 2}\right)$ replaced by $\left[2 \mathrm{~J}_{1}(\pi / 2 \sqrt{2}) /(\pi / 2 \sqrt{2})\right]\left[2 \mathrm{~J}_{0}(\pi / 2 \sqrt{2})-2 \mathrm{~J}_{1}(\pi / 2 \sqrt{2}) /(\pi / 2 \sqrt{2})\right]$ and $J_{0}\left(2 \pi \sqrt{\phi_{c}} / 3\right)$ replaced by $\left[2 J_{1}(\pi / 3) /(\pi / 3)\right]\left\{2 J_{0}(\pi / 3)-2 J_{1}(\pi / 3) /(\pi / 3)\right]$.

Comparing then again Eqs. (3.6) and (3.7) shows the error $\varphi_{\mathbf{m}}{ }^{-\gamma_{\mathbf{m}}}$ between the two output sequences. This error now takes its maximum absolute value 0.04532 for $\psi_{\mathrm{h}}=0.47833$ and $\psi_{1}=0$ in the rectangular case, and its maximum absolute value 0.06410 for $\psi_{\mathrm{h}}=0.48101$ and $\psi_{1}=0$ in the hexagonal case. When we compare these error values with the errors that we found for the optimum linearized system, we conclude that the two linearized versions are almost of the same quality.

If linear predistortion does not give sufficient error reduction, a more sophisticated way to reduce the errors must be applied. This will be the subject of the next section, where the inverse of the nonlinear system described by Eq.(2.18) will be derived. 


\section{The inverse system}

Equation (2.18) can be interpreted as the Volterra series [6-7] describing the input-output relationship of a nonlinear, discrete system. It explicitly expresses the sample values $\varphi_{\text {m }}$ of the output signal in terms of the parameter values $\psi_{m}$ of the input signal. In our case, however, the sample values of the band-limited output signal, which has to be generated, are given, and we ask for the parameters of the input signal. We can explicitly express $\psi_{\mathrm{m}}$ in $\varphi_{\mathbf{m}}$, too, if we know the Volterra series of the inverse nonlinear system. It is known [7] how the inverse Volterra series can be determined; we shall indicate how it can be constructed from the original series [Eq.(2.18)], using only the expressions for the algebraic reversion of ordinary power series $[10]$.

Let $\varphi$ be given as a power series in $\psi$ :

$\varphi=\mathrm{p}_{1} \psi+\mathrm{p}_{2} \psi^{2}+\mathrm{p}_{3} \psi^{3}+\ldots$

The problem of series reversion is to find $\psi$ as a power series in $\varphi$, i.e., to determine the coefficients $f_{r}$ in the expansion

$\psi=\mathrm{f}_{1} \varphi+\mathrm{f}_{2} \varphi^{2}+\mathrm{f}_{3} \varphi^{3}+\ldots$ 
The systematic way to do this is to write Eq. (4.1) in the form

$\psi=\frac{\varphi-\left(\mathrm{p}_{2} \psi^{2}+\mathrm{p}_{3} \psi^{3}+\ldots\right)}{\mathrm{p}_{1}}=\mathrm{q}_{1} \varphi-\left(\mathrm{q}_{2} \psi^{2}+\mathrm{q}_{3} \psi^{3}+\ldots\right)$,

where the coefficients $q_{r}(r-1,2, \ldots)$ are defined by

$\mathrm{q}_{1} \mathrm{p}_{1}=1$

$\mathrm{q}_{\mathrm{r}} \mathrm{p}_{1}=\mathrm{p}_{\mathrm{r}} \quad(\mathrm{r}=2,3, \ldots)$

and then substitute the formulas for the powers of $\psi$ :

$\psi^{1}=\mathrm{f}_{1} \varphi+\mathrm{f}_{2} \varphi^{2}+\mathrm{f}_{3} \varphi^{3} \quad+\ldots$

$\psi^{2}=\quad\left(f_{1} \varphi\right)^{2}+2\left(f_{1} \varphi\right)\left(f_{2} \varphi^{2}\right)+\ldots$

$\psi^{3}=\quad\left(\mathrm{f}_{1} \varphi\right)^{3}+\ldots$

By equating coefficients of $\varphi$ we get the formulas 


$$
\begin{aligned}
& f_{1} \varphi^{1}=q_{1} \varphi, \\
& f_{2} \varphi^{2}=-q_{2}\left(f_{1} \varphi\right)^{2}, \\
& f_{3} \varphi^{3}=-q_{3}\left(f_{1} \varphi\right)^{3}-2 q_{2}\left(f_{1} \varphi\right)\left(f_{2} \varphi^{2}\right),
\end{aligned}
$$

which determine the coefficients $f_{r}$ successively. Substituting these formulas in Eq. (4.2) and eliminating the coefficients $f_{r}$, we arrive at the expression

$\psi=\mathrm{q}_{1} \varphi-\mathrm{q}_{2}\left(\mathrm{q}_{1} \varphi\right)^{2}-\mathrm{q}_{3}\left(\mathrm{q}_{1} \varphi\right)^{3}+2 \mathrm{q}_{2}\left(\mathrm{q}_{1} \varphi\right)\left[\mathrm{q}_{2}\left(\mathrm{q}_{1} \varphi\right)^{2}\right]+\ldots . \quad(4.5)$

Formulas for power-series reversion up to a higher order are available [10].

If we now compare Eq. (4.1) and Eq. (2.18), we note that a multiplication with $\mathrm{p}_{\mathrm{r}}$ in Eq.(4.1) corresponds to a convolution with $r_{p}$ in Eq.(2.18). Analogous1y, replacing in Eq. (4.5) any multiplication with $\mathrm{q}_{r}$ by a convolution with ${ }^{\mathrm{r}_{\mathrm{q}}}$ yields the Volterra series of the inverse system 


$$
\begin{aligned}
& \psi_{\mathbf{m}}^{-\psi_{c}}=\sum_{\mathbf{k}}^{1} \mathrm{q}_{\mathbf{m}-\mathbf{k}}\left(\varphi_{\mathbf{k}}-\varphi_{\mathrm{c}}\right) \\
& -\sum_{\mathbf{k}}^{2} q_{\mathbf{m}-\mathbf{k}}\left[\sum_{\mathbf{j}}^{1} q_{\mathbf{k}-\mathbf{j}}\left(\varphi_{\mathbf{j}}-\varphi_{c}\right)\right]^{2} \\
& -\sum_{\mathbf{k}}^{3} q_{m-\mathbf{k}}\left[\sum_{\mathbf{j}}^{1}{ }^{q_{\mathbf{k}-\mathbf{j}}}\left(\varphi_{\mathbf{j}}-\varphi_{c}\right)\right]^{3} \\
& \left.\left.+2 \sum_{\mathbf{k}}^{2} q_{\mathbf{n}-\mathbf{k}}\left(\left[\sum_{\mathbf{j}}{ }^{1} q_{\mathbf{k}-\mathbf{j}}\left(\varphi_{\mathbf{j}}-\varphi_{\mathbf{c}}\right)\right]\right\} \sum_{\mathbf{j}}{ }^{2} q_{\mathbf{k}-\mathbf{j}}\left[\sum_{\mathbf{n}}{ }^{1} q_{\mathbf{j}-\mathbf{n}}\left(\varphi_{\mathbf{n}}-\varphi_{\mathbf{c}}\right)\right]^{2}\right\}\right) \\
& +\ldots
\end{aligned}
$$

The sequences ${ }^{x}{ }_{q_{k}}$ follow - via Eq. (2.14) - from their Fourier transforms ${ }^{r_{q}}\left(x^{t} u\right)$, which can be derived from the functions $r_{\bar{p}}\left(\mathbf{x}^{t} \mathbf{u}\right)$ through the relations [c.f. Eqs. (4.4)]

$$
\begin{aligned}
& { }_{\bar{q}}\left(x^{t} \mathbf{u}\right){ }^{1} \bar{p}\left(x^{t} \mathbf{u}\right)=1, \\
& r_{\bar{q}}\left(x^{t} \mathbf{u}\right){ }^{1} \bar{p}\left(x^{t} \mathbf{u}\right)={ }^{r} \bar{p}\left(x^{t} \mathbf{u}\right) \quad(r=2,3, \ldots) .
\end{aligned}
$$

Note that only the first-order term ${ }^{1} p$ needs to be invertable.

The concept of the inverse system allows us to achieve a complete compensation of the errors that occur when we want to generate a band-limited function by low-pass filtering a regular array of area-modulated pulses. Of course, the form of the 
inverse system as described by Eq.(4.6) is rather complicated, and we have to restrict the number of terms in Eq. (4.6) that we can take into account, when we apply nonlinear predistortion by means of a computer.

Let us consider which error reduction can be achieved, in the case of area-modulated circular unit-height pulses, when we apply a second-order nonlinear predistortion. Let the sample values of the band-limited function to be generated, be denoted by $\gamma_{m}$. The second-order nonlinear predistortion now takes the form

$\psi_{\mathbf{m}}=\sum_{\mathbf{k}}^{1} \mathrm{q}_{\mathbf{m}-\mathbf{k}} \gamma_{\mathbf{k}}-\sum_{\mathbf{k}}^{2} \mathrm{q}_{\mathbf{m}-\mathbf{k}}\left(\sum_{\mathbf{j}}^{1}{ }^{1} \mathrm{q}_{\mathbf{k}-\mathbf{j}} \boldsymbol{\gamma}_{\mathbf{j}}-\psi_{\mathrm{c}}\right)^{2}$,

where we have used the property that in the case of pulse-area modulation $\psi_{c}=\varphi_{c} / D$ and ${ }^{1} \dot{q}(0)=1 /{ }_{p}^{1}(0)=1 / D$. When we apply the array of alternating area values

$\gamma_{\mathbf{m}}=\frac{\gamma_{\mathrm{h}}{ }^{+\gamma} \gamma_{1}}{2}+\frac{\gamma_{h^{-\gamma}}}{2} \cos \left[\pi\left(m_{1}+m_{2}\right)\right]$

$\gamma_{\mathbf{m}}=\frac{\gamma_{h}+2 \gamma_{1}}{3}+\frac{\gamma_{h}-\gamma_{1}}{3} 2 \cos \left[\frac{2 \pi}{3}\left(m_{1}+m_{2}\right)\right]$

in the case of rectangular and hexagonal sampling, respectively, to the input of the nonlinear system described by Eq.(4.8), the array given by Eqs.(3.5) will be produced at the output of this 
system; the values $\gamma_{h}$ and $\gamma_{1}$ can be expressed explicitly in terms of the values $\psi_{\mathrm{h}}$ and $\psi_{1}$. The error between $\varphi_{\mathrm{m}}$ (the sample values of the band-1imited signal that is actually generated) and $\gamma_{m}$ (the sample values of the band-limited signal that we want to generate) follows from comparing Eqs.(3.6) and (4.9). When we substutue the values of $\psi_{h}$ and $\psi_{1}$ for which this error takes its maximum absolute value, and then minimize this maximum error with respect to $\psi_{c}$, we get an optimum for $\psi_{c}$. For rectangular sampling, we thus find the optimum value $\psi_{c}=0.38492$, in which case the error takes its maximum absolute value 0.01723 for $\psi_{h}=1$ and $\psi_{1}=0.54414$ or $\psi_{\mathrm{h}}=0.18825$ and $\psi_{1}=0$; for hexagonal sampling, the optimum value reads $\psi_{\mathrm{c}}=0.43455$, in which case the error takes its maximum absolute value 0.02051 for $\psi_{h}=1$ and $\psi_{1}=0.53335$ or $\psi_{\mathrm{h}}=0.15734$ and $\psi_{1}=0$. When we compare these errors with the errors that we found in the case of optimum first-order predistortion (0.04419 for rectangular sampling and 0.06267 for hexagonal sampling), we see that the optimum second-order predistortion gives a much better result. The coefficients ${ }^{1} q_{k}$ and ${ }^{2} q_{k}$ for these values of $\psi_{c}$ are listed in Tables $7 \mathrm{a}, \mathrm{b}$ for rectangular sampling and in Tables $8 \mathrm{a}, \mathrm{b}$ for hexagonal sampling.

We finally consider the case $\psi_{c}=0$, and investigate which error reduction can be achieved when we apply second-order predistortion, described by 
$\psi_{\mathbf{m}}=\mathrm{D}^{-1} \boldsymbol{\gamma}_{\mathbf{m}}-\mathrm{D}^{-3} \sum_{\mathbf{k}}^{2} \mathrm{p}_{\mathbf{m}-\mathbf{k}} \boldsymbol{\gamma}_{\mathbf{k}}^{2}$

and third-order predistortion, described by

$$
\begin{gathered}
\psi_{\mathbf{m}}=D^{-1} \gamma_{\mathbf{m}}-D^{-3} \sum_{\mathbf{k}}^{2} \mathrm{p}_{\mathbf{m}-\mathbf{k}} \boldsymbol{\gamma}_{\mathbf{k}}^{2}-D^{-4} \sum_{\mathbf{k}}{ }^{3} \mathrm{p}_{\mathbf{m}-\mathbf{k}} \boldsymbol{\gamma}_{\mathbf{k}}^{3}+ \\
+D^{-5} 2 \sum_{\mathbf{k}}{ }^{2} \mathrm{P}_{\mathbf{m}-\mathbf{k}}\left(\gamma_{\mathbf{k}} \sum_{\mathbf{j}}{ }^{2} \mathrm{P}_{\mathbf{k}-\mathbf{j}} \gamma_{\mathbf{j}}^{2}\right) .
\end{gathered}
$$

We apply the array of alternating area values given by Eqs. (4.9) to the input of the nonlinear correction system described by Eq. (4.10) or Eq. (4.11), and determine those values $\gamma_{\mathrm{h}}$ and $\gamma_{1}$ for which the error $\varphi_{\mathbf{m}}{ }^{-\gamma} \gamma_{\mathbf{m}}$ (the difference between the sample values of the band-limited signal that is actually generated and the sample values of the band-limited signal that we want to generate) takes its maximum absolute value under the constraint that the pulse-area parameter $\psi_{m}$ remains bounded by 0 and 1 . In the case of second-order predistortion, we thus find for rectangular sampling the maximum absolute error 0.05479 for $\gamma_{h}=0.65766$ and $\gamma_{1}=0.32749$, while for hexagonal sampling we find the maximum absolute error 0.08173 for $\gamma_{h}=0.72149$ and $\gamma_{1}=0.24607$. In the case of third-order predistortion, the maximum absolute error for rectangular sampling takes the value 0.02342 for $\gamma_{h}=0.64968$ and $\gamma_{1}=0.46144$, while for hexagonal sampling the maximum absolute error becomes 0.03620 for $\gamma_{h}=0.69187$ and 
$\gamma_{1}=0.36356$. All values for the various cases that we have considered are collected in Table 9 a for rectangular sampling and in Table $9 \mathrm{~b}$ for hexagonal sampling. We may conclude that the quality of the predistortion scheme increases with increasing complexity of the predistortion algorithm: 1st order (no correction), optimum or Tschebyscheff 1st order, optimum 2nd order; note that in order to obtain a certain error reduction, the optimum predistortion schemes have a lower complexity than the schemes for which $\psi_{c}=0$. More complex, higher-order predistorion schemes are necessary, of course, if a better error reduction is required; such higher-order schemes can be found along the lines described in this report.

When we want to use predistortion, we must restrict the values $\gamma_{m}$ to a certain range $\gamma_{\min } \leq \gamma \leq \gamma_{\max }$. This range should be such that the resulting pulse areas $\psi_{\mathrm{m}}$ are in the range $0 \leq \psi \leq 1$; hence, negative pulse areas and overlapping pulses are avoided. Finding the maximum value of $\gamma_{\max }$ and the minimum value of $\gamma_{\min }$ (yielding the largest range $\gamma_{\max }-\gamma_{\min }$ ) such that for a11 situations the condition $0 \leq \psi \leq 1$ is satisfied, is difficult. To get an indication of $\gamma_{\max }$ and $\gamma_{\min }$, we apply as input array to the predistortion system, an array $\gamma_{m}$ whose values alternate between $\gamma_{\max }$ and $\gamma_{\min }$ [as in Eqs. (4.9), with $\gamma_{h}=\gamma_{\max }$ and $\gamma_{1}=\gamma_{\min }$ ], and find those values of $\gamma_{\max }$ and $\gamma_{\min }$ for which the system yields pulse areas $\psi_{m}$ that alternate between 1 and 0 [as in Eqs.(3.5), with $\psi_{h}=1$ and $\psi_{1}=0$ l. These values of $\gamma_{\max }$ and $\gamma_{\min }$, and the 
range $\gamma_{\max }{ }^{-\gamma} \min _{\text {m }}$, for the various predistortion schemes have been collected in Table 10a for rectangular sampling and in Table 10b for hexagonal sampling. We expect that for rectangular sampling, the optimum values of $\gamma_{\max }$ and $\gamma_{\min }$ will be close to the values in Table 10a, since the alternating array [Eq.(3.5)] of pulse areas $\psi_{\mathrm{m}}$ has a $\psi_{\mathrm{h}}$ vs. $\psi_{1}$ distribution that resembles the sign distribution of the coefficient arrays ${ }^{r} q_{k}$ and $g_{k}$; this is not the case for hexagonal sampling. Hence, we may expect that in the case of hexagonal sampling, the range will be somewhat smaller, with a lower value of $\gamma_{\max }$ and a higher value of $\gamma_{\min }$ than the values presented in Table $10 \mathrm{~b}$. 


\section{Application to computer-generated transparencies}

The technique of predistortion as described above, can be used in the synthesis of coherent optical fields, where a computer-generated transparency whose transparency function is given by $\psi(\mathbf{x})$ [c.f. Eq.(2.6)] is illuminated by a plane wave of monochromatic laser light, and the light behind the transparency is low-pass filtered to construct the band-limited amplitude distribution $\varphi(x)$ [c.f. Eq.(2.5)] in the output plane (see Fig.5). In many practical situations the computer-generated transparency will be a half-tone transparency consisting of, for instance, fully transparent circular pulses on an opaque background; in this case we can use the formulas derived in this report. Using predistortion in computer-generating half-tone transparencies, we can extend the dynamical range of the transparency, since we are no longer limited to narrow pulses in order to avoid distortion.

If we use a half-tone transparency in the set-up of Fig.5, we can, of course, only synthesize real and positive light amplitudes. Moreover, to avoid negative pulse areas and overlapping pulses, the sample values $\gamma_{m}$ that we want to generate, must be restricted to the range $\gamma_{\min } \leq \gamma \leq \gamma_{\max }$. The upper bound does not present a severe problem; it requires a mere scaling of the light amplitude. The lower bound, however, cannot 
be solved that easily. If the required sample values $\gamma_{\mathrm{m}}$ extend below $\gamma_{\min }$, negative pulse areas may arise. The best we can do in that case seems to replace a pulse having a negative area by a pulse having zero area; we must realize that an error will then occur. The problem of negative pulse areas can be solved completely, if we are allowed to properly modify the function that we want to synthesize and bring the required sample values within the necessary range. Such a modification is permitted in the important case of computer holography.

Computer holography [2-3] enables us to realize a negative real or complex band-limited light amplitude $\varphi_{\text {complex }}(\mathbf{x})$, say, whose Fourier transform $\dot{\varphi}_{\text {complex }}(\mathbf{u})$ vanishes outside the interval $\mathbf{u} \in \mathbf{\Omega}_{c}$, by means of a transparency that can itself, if used in the set-up of Fig.5, realize the positive function

$\varphi(\mathbf{x})=\mathrm{b}(\mathbf{x})+2 \operatorname{Re}\left\{\varphi_{\text {complex }}(\mathbf{x}) \exp \left[i u_{0}^{t} x\right]\right\}$

The additional bias function $b(x)$ allows us to construct $\varphi(x)$ in such a way that no negative pulse areas will occur in the transparency. If the modulating frequency $u_{0}$ is chosen properly, the Fourier transform

$\bar{\varphi}(\mathbf{u})=\bar{b}(\mathbf{u})+\bar{\varphi}_{\text {complex }}\left(\mathbf{u}-\mathbf{u}_{\mathrm{o}}\right)+\bar{\varphi}_{\text {complex }}^{-*}\left(\mathbf{u}+\mathbf{u}_{\mathrm{o}}\right)$

consists of three non-overlapping components. By blocking in the 


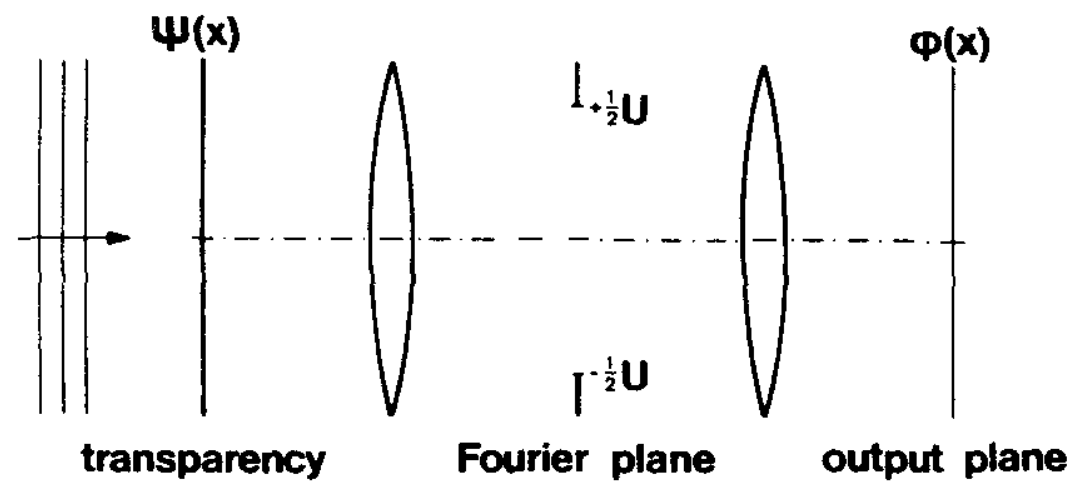

Figure 5. Synthesis of coherent optical fields.

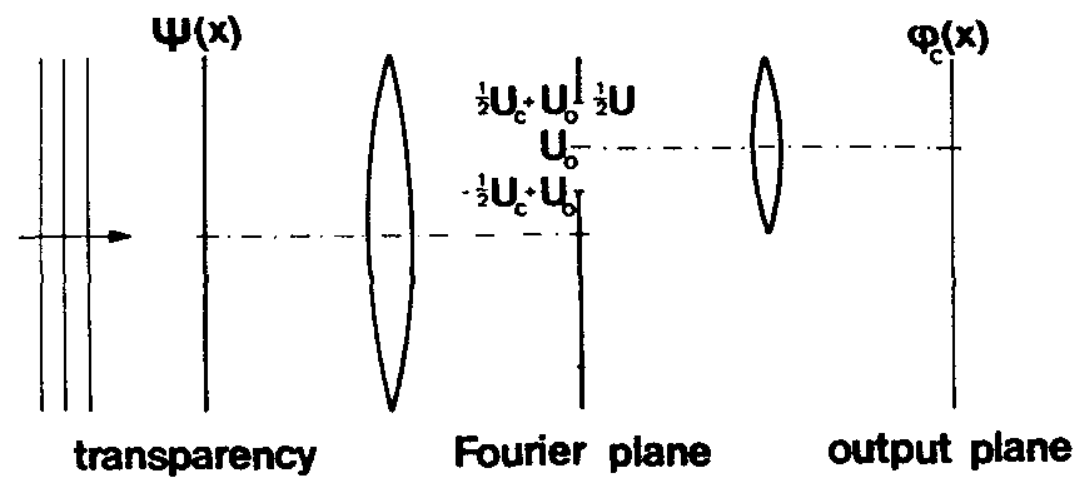

Figure 6. Synthesis of coherent optical fields using holographic concepts. 


\begin{abstract}
Fourier plane all components except $\bar{\varphi}_{\text {complex }}\left(\mathbf{u}-\mathbf{u}_{\mathrm{o}}\right)$ and shifting the optical axis over a distance $\mathbf{u}_{0}$, the complex amplitude $\varphi_{\text {complex }}(\mathbf{x})$ will occur in the output plane (see Fig.6). In the case of computer holography, our transparency resembles the computer hologram of Burch [11] rather than Lohmann's detour-phase hologram [12]. In fact, predistortion as described in this report cannot be applied to the Lohmann-type hologram, since in this type of hologram the pulses are not equally spaced. Nevertheless, the method of predistortion outlined in this report, allows us to synthesize band-limited complex light amplitudes to any degree of accuracy.
\end{abstract}




\section{References}

[1] Papoulis, A.

SYSTEMS AND TRANSFORMS WITH APPLICATIONS IN OPTICS.

New York: McGraw-Hill, 1968.

McGraw-Hill series in systems science. P. 119-128.

[2] Lee, W. - H.

COMPUTER-GENERATED HOLOGRAMS: Techniques and applications.

In: Progress in Optics, Vol.16. Ed. by E. Wolf.

Amsterdam: North-Holland, 1978. P. 119-232.

[3] Dallas, W.J.

COMPUTER - GENERATED HOLOGRAMS .

In: The Computer in Optical Research: Methods and

applications. Ed. by B.R. Frieden.

Berlin: Springer, 1980.

Topics in applied physics, Vol. 41. P. 291-366.

[4] Bastiaans, M.J.

A GENERALIZED SAMPLING THEOREM WITH APPLICATION TO COMPUTER-GENERATED TRANSPARENCIES .

J. Opt. Soc. Am., Vol. 68 (1978), p. 1658-1665.

[5] Dudgeon, D.E. and R.M. Mersereau

MULTIDIMENSIONAL DIGITAL SIGNAL PROCESSING.

Englewood Cliffs, N.J.: Prentice-Ha11, 1984.

Prentice-Hall signal processing series.

[6] Volterra, V.

THEORY OF FUNCTIONALS AND OF INTEGRALS AND

INTEGRO-DIFFERENTIAL EQUATIONS.

New York: Dover, 1959.

[7] Halme, A.

POLYNOMIAL OPERATORS FOR NONLINEAR SYSTEMS ANALYSIS.

Doctor of Technology Thesis. Helsinki University of

Technology, 1972.

Acta Polytechn. Scand. Math. \& Comput. Mach. Ser., No. 24.

[8] HANDBOOK OF MATHEMATICAL FUNCTIONS. Ed. by M. Abramowitz and I.A. Stegun.

New York: Dover, 1970. Chapter 22.

[9] Gradshteyn, I.S. and I.M. Ryzhik

TABLE OF INTEGRALS, SERIES, AND PRODUCTS.

New York: Academic Press, 1965. Eq. 6.681.1.

[10] Pipes, L.A.

THE REVERSION METHOD FOR SOLVING NONLINEAR DIFFERENTIAL EQUATIONS .

J. Appl. Phys., Vol. 23 (1952), p. 202-207.

[11] Burch, J.J.

A COMPUTER ALGORITHM FOR THE SYNTHESIS OF SPATIAL FREQUENCY FILTERS.

Proc. IEEE, Vol. 55 (1967), p. 599-601.

[12] Brown, B.R. and A.W. Lohmann

COMPLEX SPATIAL FILTERING WITH BINARY MASKS.

Appl. Opt., Vol. 5 (1966), p. 967-969. 


\begin{tabular}{|l|rrrrrr|}
\hline$k_{2} \backslash k_{1}$ & 0 & 1 & 2 & 3 & 4 & 5 \\
\hline 0 & -.16149 & & & & & \\
1 & .04909 & 0 & & & & \\
2 & -.01227 & 0 & 0 & & & \\
3 & .00545 & 0 & 0 & 0 & & \\
4 & -.00307 & 0 & 0 & 0 & 0 & \\
5 & .00196 & 0 & 0 & 0 & 0 & 0 \\
6 & -.00136 & 0 & 0 & 0 & 0 & 0 \\
7 & .00100 & 0 & 0 & 0 & 0 & 0 \\
8 & -.00077 & 0 & 0 & 0 & 0 & 0 \\
9 & .00061 & 0 & 0 & 0 & 0 & 0 \\
10 & -.00049 & 0 & 0 & 0 & 0 & 0 \\
\hline
\end{tabular}

Table la. Coefficients ${ }^{2} \mathrm{p}_{\mathbf{k}}$ for $\psi_{\mathrm{c}}=0$ in the rectangular case

\begin{tabular}{|l|rrrrrr|}
\hline$k_{2} \backslash k_{1}$ & \multicolumn{1}{|c}{0} & 1 & 2 & 3 & 4 & \multicolumn{1}{c|}{5} \\
\hline 0 & .01550 & & & & & \\
1 & -.00732 & .00205 & & & & \\
2 & .00298 & -.00051 & .00013 & & & \\
3 & -.00142 & .00023 & -.00006 & .00003 & & \\
4 & .00082 & -.00013 & .00003 & -.00001 & .00001 & \\
5 & -.00053 & .00008 & -.00002 & .00001 & -.00001 & .00000 \\
6 & .00037 & -.00006 & .00001 & -.00001 & .00000 & -.00000 \\
7 & -.00027 & .00004 & -.00001 & .00000 & -.00000 & .00000 \\
8 & .00021 & -.00003 & .00001 & -.00000 & .00000 & -.00000 \\
9 & -.00017 & .00003 & -.00001 & .00000 & -.00000 & .00000 \\
10 & .00013 & -.00002 & .00001 & -.00000 & .00000 & -.00000 \\
\hline
\end{tabular}

Table 1b. Coefficients ${ }^{3} \mathrm{p}_{\mathbf{k}}$ for $\psi_{\mathrm{c}}=0$ in the rectangular case 


\begin{tabular}{|l|rrrrrr|}
\hline$k_{2} \backslash k_{1}$ & \multicolumn{1}{|c}{0} & 1 & 2 & \multicolumn{1}{c}{3} & \multicolumn{1}{c}{4} & 5 \\
\hline 0 & -.20719 & & & & & \\
1 & .04687 & & & & & \\
2 & -.00586 & -.01260 & & & & \\
3 & .00000 & .00469 & & & & \\
4 & .00073 & -.00134 & -.00315 & & & \\
5 & -.00037 & .00000 & .00167 & & & \\
6 & .00000 & .00030 & -.00059 & -.00140 & & \\
7 & .00014 & -.00018 & .00000 & .00085 & & \\
8 & -.00009 & .00000 & .00017 & -.00033 & -.00079 & \\
9 & .00000 & .00008 & -.00011 & .00000 & .00051 & \\
10 & .00005 & -.00006 & .00000 & .00011 & -.00021 & -.00050 \\
\hline
\end{tabular}

Table 2a. Coefficients ${ }^{2} \mathrm{p}_{\mathbf{k}}$ for $\psi_{\mathrm{c}}=0$ in the hexagonal case

\begin{tabular}{|l|rrrrrr|}
\hline$k_{2} \backslash k_{1}$ & \multicolumn{1}{c}{0} & 1 & 2 & 3 & 4 & 5 \\
\hline 0 & .02121 & & & & & \\
1 & -.00664 & & & & & \\
2 & .00115 & .00360 & & & & \\
3 & .00017 & -.00135 & & & & \\
4 & -.00028 & .00035 & .00094 & & & \\
5 & .00012 & .00005 & -.00049 & & & \\
6 & .00001 & -.00012 & .00016 & .00042 & & \\
7 & -.00005 & .00006 & .00002 & -.00025 & & \\
8 & .00003 & .00000 & -.00006 & .00009 & .00024 & \\
9 & .00000 & -.00003 & .00004 & .00001 & -.00015 & \\
10 & -.00002 & .00002 & .00000 & -.00004 & .00006 & .00015 \\
\hline
\end{tabular}

Table 2b. Coefficients ${ }^{3} p_{k}$ for $\psi_{c}=0$ in the hexagonal case 


\begin{tabular}{|c|rrrrrr|}
\hline$k_{2} \backslash k_{1}$ & \multicolumn{1}{|c}{0} & 1 & 2 & 3 & \multicolumn{1}{c|}{4} & \multicolumn{1}{c|}{5} \\
\hline 0 & .64299 & & & & & \\
1 & .04164 & .00127 & & & & \\
2 & -.00970 & -.00030 & .00007 & & & \\
3 & .00426 & .00013 & -.00003 & .00001 & & \\
4 & -.00238 & -.00007 & .00002 & -.00001 & .00000 & \\
5 & .00152 & .00005 & -.00001 & .00001 & -.00000 & .00000 \\
6 & -.00106 & -.00003 & .00001 & -.00000 & .00000 & -.00000 \\
7 & .00078 & .00002 & -.00001 & .00000 & -.00000 & .00000 \\
8 & -.00059 & -.00002 & .00000 & -.00000 & .00000 & -.00000 \\
9 & .00047 & .00001 & -.00000 & .00000 & -.00000 & .00000 \\
10 & -.00038 & -.00001 & .00000 & -.00000 & .00000 & -.00000 \\
\hline
\end{tabular}

Table 3a. Coefficients ${ }^{1} \mathrm{p}_{\mathbf{k}}$ for $\psi_{\mathrm{c}}-0.47183$ in the rectangular case.

\begin{tabular}{|l|rrrrrr|}
\hline$k_{2} \backslash k_{1}$ & \multicolumn{1}{|c}{0} & 1 & 2 & 3 & \multicolumn{1}{c}{4} & \multicolumn{1}{c|}{5} \\
\hline 0 & 1.58587 & & & & & \\
1 & -.10834 & .01164 & & & & \\
2 & .03345 & -.00390 & .00133 & & & \\
3 & -.01574 & .00188 & -.00065 & .00032 & & \\
4 & .00906 & -.00110 & .00038 & -.00019 & .00011 & \\
5 & -.00586 & .00072 & -.00025 & .00012 & -.00007 & .00005 \\
6 & .00410 & -.00050 & .00017 & -.00009 & .00005 & -.00003 \\
7 & -.00302 & .00037 & -.00013 & .00006 & -.00004 & .00002 \\
8 & .00232 & -.00029 & .00010 & -.00005 & .00003 & -.00002 \\
9 & -.00184 & .00023 & -.00008 & .00004 & -.00002 & .00001 \\
10 & .00149 & -.00018 & .00006 & -.00003 & .00002 & -.00001 \\
\hline
\end{tabular}

Table $3 b$. Coefficients ${ }_{q_{k}}$ for $\psi_{c}=0.47183$ in the rectangular case. 


\begin{tabular}{|l|rrrrrr|}
\hline$k_{2} \backslash k_{1}$ & \multicolumn{1}{c}{0} & 1 & 2 & 3 & 4 & 5 \\
\hline 0 & .72383 & & & & & \\
1 & .04024 & & & & & \\
2 & -.00482 & -.00967 & & & & \\
3 & .00010 & .00360 & & & & \\
4 & .00052 & -00105 & -.00240 & & & \\
5 & -.00028 & .00003 & .00128 & & & \\
6 & .00001 & .00022 & -.00046 & -.00106 & & \\
7 & .00010 & -.00013 & .00001 & .00065 & & \\
8 & -.00007 & .00000 & .00012 & -.00026 & -.00060 & \\
9 & .00000 & .00006 & -.00008 & .00001 & .00039 & \\
10 & .00003 & -.00004 & .00000 & .00008 & -.00016 & -.00038 \\
\hline
\end{tabular}

Table 4a. Coefficients ${ }^{1} p_{k}$ for $\psi_{c}=0.47522$ in the hexagonal case.

\begin{tabular}{|l|rrrrrr|}
\hline$k_{2} \backslash k_{1}$ & 0 & 1 & 2 & 3 & 4 & 5 \\
\hline 0 & 1.41080 & & & & & \\
1 & -.07653 & & & & & \\
2 & .01028 & .02818 & & & & \\
3 & .00115 & -.01077 & & & & \\
4 & -.00216 & .00281 & .00759 & & & \\
5 & .00094 & .00033 & -.00400 & & & \\
6 & .00009 & -.00092 & .00128 & .00343 & & \\
7 & -.00042 & .00047 & .00016 & -.00206 & & \\
8 & .00025 & .00004 & -.00051 & .00072 & .00194 & \\
9 & .00002 & -.00024 & .00028 & .00009 & -.00125 & \\
10 & -.00014 & .00015 & .00002 & -.00032 & .00047 & .00125 \\
\hline
\end{tabular}

Table 4b. Coefficients ${ }^{1} q_{k}$ for $\psi_{c}=0.47522$ in the hexagonal case. 


\begin{tabular}{|c|rrrrrr|}
\hline$k_{2} \backslash k_{1}$ & 0 & 1 & 2 & 3 & 4 & 5 \\
\hline 0 & .63769 & & & & & \\
1 & .04266 & .00171 & & & & \\
2 & -.00969 & -.00040 & .00009 & & & \\
3 & .00424 & .00018 & -.00004 & .00002 & & \\
4 & -.00237 & -.00010 & .00002 & -.00001 & .00001 & \\
5 & .00151 & .00006 & -.00001 & .00001 & -.00000 & .00000 \\
6 & -.00105 & -.00004 & .00001 & -.00000 & .00000 & -.00000 \\
7 & .00077 & .00003 & -.00001 & .00000 & -.00000 & .00000 \\
8 & -.00059 & -.00002 & .00001 & -.00000 & .00000 & -.00000 \\
9 & .00047 & .00002 & -.00000 & .00000 & -.00000 & .00000 \\
10 & -.00038 & -.00002 & .00000 & -.00000 & .00000 & -.00000 \\
\hline
\end{tabular}

Table 5a. Coefficients $h_{k}$ in the rectangular case.

\begin{tabular}{|l|rrrrrr|}
\hline$k_{2} \backslash k_{1}$ & 0 & 1 & 2 & 3 & 4 & 5 \\
\hline 0 & 1.60097 & & & & & \\
1 & -.11277 & .01139 & & & & \\
2 & .03443 & -.00372 & .00123 & & & \\
3 & -.01612 & .00178 & -.00059 & .00029 & & \\
4 & .00926 & -.00103 & .00035 & -.00017 & .00010 & \\
5 & -.00598 & .00067 & -.00022 & .00011 & -.00006 & .00004 \\
6 & .00418 & -.00047 & .00016 & -.00008 & .00004 & -.00003 \\
7 & -.00308 & .00035 & -.00012 & .00006 & -.00003 & .00002 \\
8 & .00236 & -.00027 & .00009 & -.00004 & .00003 & -.00002 \\
9 & -.00187 & .00021 & -.00007 & .00003 & -.00002 & .00001 \\
10 & .00152 & -.00017 & .00006 & -.00003 & .00002 & -.00001 \\
\hline
\end{tabular}

Table 5b. Coefficients $\mathbf{g}_{\mathbf{k}}$ in the rectangular case. 


\begin{tabular}{|l|rrrrrr|}
\hline$k_{2} \backslash k_{1}$ & 0 & 1 & 2 & 3 & 4 & 5 \\
\hline 0 & .71855 & & & & & \\
1 & .04102 & & & & & \\
2 & -.00483 & -.00949 & & & & \\
3 & .00013 & .00354 & & & & \\
4 & .00050 & -00104 & -.00235 & & & \\
5 & -.00027 & .00003 & .00125 & & & \\
6 & .00001 & .00021 & -.00045 & -.00104 & & \\
7 & .00009 & -.00013 & .00002 & .00064 & & \\
8 & -.00007 & .00000 & .00012 & -.00025 & -.00059 & \\
9 & .00000 & .00005 & -.00008 & .00001 & .00039 & \\
10 & .00003 & -.00004 & .00000 & .00007 & -.00016 & -.00037 \\
\hline
\end{tabular}

Table 6a. Coefficients $h_{k}$ in the hexagonal case.

\begin{tabular}{|c|rrrrrr|}
\hline$k_{2} \backslash k_{1}$ & 0 & 1 & 2 & 3 & 4 & 5 \\
\hline 0 & 1.42250 & & & & & \\
1 & -.07902 & & & & & \\
2 & .01062 & .02858 & & & & \\
3 & .00106 & -.01089 & & & & \\
4 & -.00213 & .00287 & .00763 & & & \\
5 & .00095 & .00030 & -.00402 & & & \\
6 & .00008 & -.00090 & .00130 & .00344 & & \\
7 & -.00041 & .00047 & .00014 & -.00207 & & \\
8 & .00025 & .00004 & -.00050 & .00073 & .00194 & \\
9 & .00002 & -.00024 & .00028 & .00008 & -.00125 & \\
10 & -.00014 & .00015 & .00002 & -.00032 & .00047 & .00125 \\
\hline
\end{tabular}

Table $6 \mathrm{~b}$. Coefficients $g_{\mathbf{k}}$ in the hexagonal case. 


\begin{tabular}{|l|rrrrrr|}
\hline$k_{2} \backslash k_{1}$ & 0 & 1 & 2 & 3 & 4 & 5 \\
\hline 0 & 1.51606 & & & & & \\
1 & -.08186 & .00689 & & & & \\
2 & .02425 & -.00218 & .00070 & & & \\
3 & -.01125 & .00103 & -.00033 & .00016 & & \\
4 & .00643 & -.00060 & .00019 & -.00009 & .00005 & \\
5 & -.00415 & .00039 & -.00012 & .00006 & -.00003 & .00002 \\
6 & .00290 & -.00027 & .00009 & -.00004 & .00002 & -.00002 \\
7 & -.00213 & .00020 & -.00006 & .00003 & -.00002 & .00001 \\
8 & .00164 & -.00015 & .00005 & -.00002 & .00001 & -.00001 \\
9 & -.00129 & .00012 & -.00004 & .00002 & -.00001 & .00001 \\
10 & .00105 & -.00010 & .00003 & -.00002 & .00001 & -.00001 \\
\hline
\end{tabular}

Table 7a. Coefficients ${ }^{1} \mathrm{q}_{\mathbf{k}}$ for $\psi_{\mathrm{c}}=0.38492$ in the rectangular case.

\begin{tabular}{|l|rrrrrr|}
\hline $\mathrm{k}_{2} \backslash \mathrm{k}_{1}$ & \multicolumn{1}{|c}{0} & 1 & 2 & 3 & \multicolumn{1}{c}{4} & 5 \\
\hline 0 & -.23340 & & & & & \\
1 & .07633 & -.00459 & & & & \\
2 & -.02161 & .00144 & -.00046 & & & \\
3 & .00991 & -.00068 & .00022 & -.00010 & & \\
4 & -.00565 & .00039 & -.00013 & .00006 & -.00003 & \\
5 & .00364 & -.00025 & .00008 & -.00004 & .00002 & -.00001 \\
6 & -.00253 & .00018 & -.00006 & .00003 & -.00002 & .00001 \\
7 & .00187 & -.00013 & .00004 & -.00002 & .00001 & -.00001 \\
8 & -.00143 & .00010 & -.00003 & .00002 & -.00001 & .00001 \\
9 & .00113 & -.00008 & .00003 & -.00001 & .00001 & -.00000 \\
10 & -.00092 & .00006 & -.00002 & .00001 & -.00001 & .00000 \\
\hline
\end{tabular}

Table $7 \mathrm{~b}$. Coefficients ${ }^{2} \mathrm{q}_{\mathbf{k}}$ for $\psi_{\mathrm{c}}=0.38492$ in the rectangular case. 


\begin{tabular}{|l|rrrrrr|}
\hline$k_{2} \backslash k_{1}$ & \multicolumn{1}{|c}{0} & 1 & 2 & 3 & 4 & 5 \\
\hline 0 & 1.37753 & & & & & \\
1 & -.06767 & & & & & \\
2 & .00907 & .02423 & & & & \\
3 & .00087 & -.00922 & & & & \\
4 & -.00180 & .00244 & .00646 & & & \\
5 & .00080 & .00025 & -.00341 & & & \\
6 & .00007 & -.00076 & .00110 & .00291 & & \\
7 & -.00034 & .00040 & .00012 & -.00175 & & \\
8 & .00021 & .00003 & -.00042 & .00062 & .00165 & \\
9 & .00001 & -.00020 & .00024 & .00007 & -.00106 & \\
10 & -.00012 & .00013 & .00002 & -.00027 & .00040 & .00106 \\
\hline
\end{tabular}

Table 8a. Coefficients ${ }^{1} q_{k}$ for $\psi_{c}=0.43455$ in the hexagonal case.

\begin{tabular}{|c|rrrrrr|}
\hline$k_{2} \backslash k_{1}$ & \multicolumn{1}{|c}{0} & \multicolumn{1}{c}{1} & \multicolumn{1}{c}{2} & \multicolumn{1}{c|}{3} & \multicolumn{1}{c|}{4} & 5 \\
\hline 0 & -.26691 & & & & & \\
1 & .06413 & & & & & \\
2 & -.00845 & -.02135 & & & & \\
3 & -.00059 & .00808 & & & & \\
4 & .00150 & -.00218 & -.00561 & & & \\
5 & -.00069 & -.00017 & .00296 & & & \\
6 & -.00005 & .00063 & -.00098 & -.00252 & & \\
7 & .00029 & -.00034 & -.00008 & .00152 & & \\
8 & -.00018 & -.00002 & .00035 & -.00055 & -.00142 & \\
9 & -.00001 & .00017 & -.00020 & -.00005 & .00092 & \\
10 & .00010 & -.00011 & -.00001 & .00022 & -.00035 & -.00091 \\
\hline
\end{tabular}

Table $8 \mathrm{~b}$. Coefficients ${ }^{2} \mathrm{q}_{\mathbf{k}}$ for $\psi_{\mathrm{c}}=0.43455$ in the hexagonal case. 


\begin{tabular}{|l|c|c|cccc|}
\hline correction & c.f. & $\psi_{\mathrm{c}}$ & $\gamma_{\mathrm{h}} \gamma_{1}$ & $\psi_{\mathrm{h}} \psi_{1}$ & $\varphi_{\mathrm{h}} \varphi_{1}$ & error \\
\hline 1st order & $\psi(4=\gamma / \mathrm{D}$ & 0 & .78540 & 1 & .58800 & .19740 \\
& & & 0 & 0 & .19740 & \\
2nd order & $(4.10)$ & 0 & .65766 & 1 & .60287 & .05479 \\
& & & .32749 & .25434 & .38228 & \\
3rd order & $(4.11)$ & 0 & .64968 & 1 & .62626 & .02342 \\
& & & .46144 & .41472 & .48486 & \\
Tschebyscheff & $(3.15)$ & - & .28012 & .47833 & .32544 & .04532 \\
1st order & & & .09556 & 0 & .05024 & \\
optimum & $(3.9)$ & .47183 & .68114 & 1 & .63695 & .04419 \\
1st order & & & .47483 & .47183 & .51903 & \\
& & .27744 & .47183 & .32163 & \\
& & & .09314 & 0 & .04895 & \\
optimum & $(4.8)$ & .38492 & .66938 & 1 & .65214 & .01723 \\
2nd order & & & .54339 & .54414 & .56062 & \\
& & & .15682 & .18825 & .13959 & \\
& & & .00897 & 0 & .00826 & \\
\hline
\end{tabular}

Table 9a. Errors arising with alternating arrays for rectangular sampling. 


\begin{tabular}{|l|c|c|cccc|}
\hline correction & c.f. & $\psi_{\mathrm{c}}$ & $\gamma_{\mathrm{h}} \gamma_{1}$ & $\psi_{\mathrm{h}} \psi_{1}$ & $\varphi_{\mathrm{h}} \varphi_{1}$ & error \\
\hline 1st order & $\psi=\gamma / \mathrm{D}$ & 0 & .90690 & 1 & .63057 & .27633 \\
& & & 0 & 0 & .13816 & \\
2nd order & $(4.10)$ & 0 & .72149 & 1 & .63976 & .08173 \\
& & & .24607 & .16911 & .28693 & \\
3rd order & $(4.11)$ & 0 & .69187 & 1 & .65567 & .03620 \\
& & & .36356 & .28233 & .38166 & \\
Tschebyscheff & $(3.15)$ & - & .30187 & .48101 & .36597 & .06410 \\
1st order & & & .06718 & 0 & .03513 & \\
optimum & $(3.9)$ & .47522 & .76189 & 1 & .69922 & .06267 \\
1st order & & & .50348 & .47522 & .53482 & \\
& & .29966 & .47522 & .36233 & \\
& & & .06566 & 0 & .03432 & \\
optimum & $(4.8)$ & .43455 & .73664 & 1 & .71613 & .02051 \\
2nd order & & .56883 & .53335 & .57908 & \\
& & & .15523 & .15734 & .13472 & \\
& & & -.00627 & 0 & .00399 & \\
\hline
\end{tabular}

Table 9b. Errors arising with alternating arrays for hexagonal sampling. 


\begin{tabular}{|l|l|ccc|}
\hline correction & c.f. & $\gamma_{\min }$ & $\gamma_{\max }$ & range \\
\hline 1st order & $\psi-\gamma / \mathrm{D}$ & 0 & .78540 & .78540 \\
2nd order & $(4.10)$ & .14982 & .63558 & .48576 \\
3rd order & $(4.11)$ & .18529 & .60011 & .41482 \\
Tschebyscheff & $(3.15)$ & .19977 & .58563 & .38586 \\
opt.1st order & $(3.9)$ & .19740 & .58800 & .39060 \\
opt.2nd order & $(4.8)$ & .20200 & .58339 & .38139 \\
\hline
\end{tabular}

Table 10a. Sample values $\gamma_{\max }$ and $\gamma_{\text {min }}$ for which the pulse areas take the values 1 and 0 , for rectangular sampling.

\begin{tabular}{|l|l|ccc|}
\hline correction & c.f. & $\gamma_{\min }$ & $\gamma_{\max }$ & range \\
\hline 1st order & $\psi=\gamma / \mathrm{D}$ & 0 & .90690 & .90690 \\
2nd order & $(4.10)$ & .09919 & .70852 & .60933 \\
3rd order & $(4.11)$ & .12290 & .66109 & .53819 \\
Tschebyscheff & $(3.15)$ & .13965 & .62759 & .48794 \\
opt.1st order & $(3.9)$ & .13816 & .63057 & .49241 \\
opt.2nd order & $(4.8)$ & .14054 & .62582 & .48528 \\
\hline
\end{tabular}

Table 10b. Sample values $\gamma_{\max }$ and $\gamma_{\min }$ for which the pulse areas take the values 1 and 0 , for hexagonal sampling. 


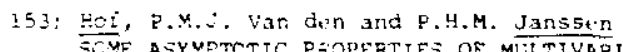
SCYE ASYMPTCTIC PÄOPERTIES OF MULTIVARIABLE MODELS IDENTIFIED BY EQUATION ERROR TECHNIQUES. E'T Report 85-E-153, 1985. ISBN 90-6144-153-6

12541 Gearlings, J.H.T. EIMIT CICEES IN DIGITAL EILTERS: A bibllography 1975-1984. EUT Report 85-E-154. i965. ISBN 90-6144-154-4

(255) 3ruxt. 3.t. G. de FI!E INFLCENCE OF A HIGH-INOEX MICHO-LENS IN A LASER-TAPER COUPLING. ELT Report 85-E-155, 1965. ISBN 90-6144-155-2

(i56) Amelsfor:, A.M.J. van and Th. Scharten A FHEORE' ILAL STUEY OF THE ELECTROMAGNETIC FIELD IN A LIMB, EXCITED EY ARTIFICIAL SOURCES. EUT Report BE-E-:56. 1У86. ISBN 90-6144-156-0

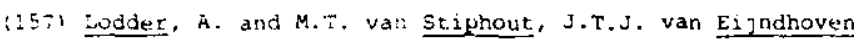
ESCHER: Elndhover. SCHentate EditoR reterence manual. EU: Report 86-E-157.:986. ISBN 90-6144-157-9

(:5s) Arnbak, J.C. DEVELOPMENT OF TRANSMISSION FACILITIES FOR ELECTRONIC MEDIA IN THE NETHERLANDS. EUT Report $86-\varepsilon-158$. 1986. ISBN 90-6144-158-?

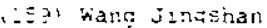

TARMONL AND RECTAAGCLAF PLLSE RE'

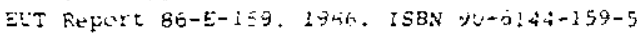

126:) Wolzzk, S.G. and A,M.F.t. Yan de Laar, E.F. Bternnis

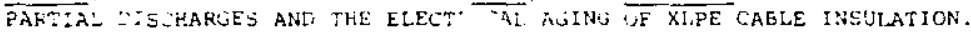

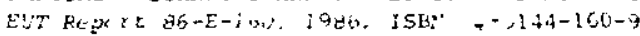

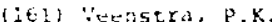

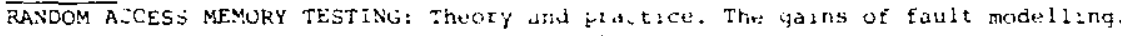
EUT Report 86-E-16́1. 1986. ISBN )-41+4-161-7

(16:) Yeer, A. $2 . \bar{F}$, vat:

TMST2OLQ EVALLAT:ON MODLLE CONTROLLER

ELT Repor: Só-E-í2. 1930. ISBN 90-6:44-162-5

(16j) छtok, L. and R. Van den gorn, G.L.t.M. Janssen HIGHER LEVELS OF A SILICON COMPILER.

EUT Report 80-E-iij3. 1986. ISBN 90-614i-163-3

(164) Engelshoten, R.J. van and J.F.M. Theoenwen GENERATING LAYOUTS FOR RANDCM LOGIC: Ceil qeneration schemes. EU? Report 36-E-164. 19EG. ISBN 90-6144-164-1

(165) ingens, P.E.in. ard A.fi.j. Slenter GADL: A gate irtay jescription Laiduatyes. EUT Repert $37-E-165,1967$. ISBN 90-53144-165-X

(16o) Dielen, M. and $\dot{F} . \mathrm{Y}$. Thereuwen AN OPT IMAL CMOS STRUC UURE FOR THE DESIGN OF A CELL LIBRARY. Eur Report 87-Ë-166. 1387. ISBN 90-1,144-156-8

(i6-) Deriemans, U.A.M. and J.F.M. Thereuwen ESKISS: A progran for optimal state assignment. EUT Report $67-E-167$. 1987. ISgN 90-6144-167-6

(í́) Linnaria, J.P.A.G.

SPATIA. DISTRIBLTION OF TRAFEIC IN A CELLULAR MOBILE DATA NETWORK. SUT Report s7-E-168. 198\%. ISBN 90-6́144-168-4

(16y) Vlack, A.J. and Plnedd de Gyvez, K.A. Post IMPLEMENTATION AND EVRLUATION OF A COMBINED TEST-ERROR CORRECTION PROCEDURE FOR MEMORIES WITH DEFECTS EUT Report 87-E-169. 1487. ISBN 90-6144-169-2

(170) Hoii ¥ا2

DASM: A tool for aecominosition and analysis of sequential machines. EUT Report $B 7-E-170$. 1987. ISBN $90-6144-170-6$

(171) Monree, P, and M.H.A.J. Herben MLLTIPLE-BEAM GROUNDSTATION REFLECTOR ANTENIA SYSTEM: A preliminary study. EUT R+? ort 87-E-171. 1987. ISBN 40-6́144-171-4

(1-2) Bastiaans, K.j. and $\mathrm{A}, \mathrm{H}, \mathrm{M}$. Akkermuns ERROR KELIUCTICN IN TWO-DIMENSIONAL PULSE-AREA MUDULATION, WITH APPLICATION TO COMPUTER-GENERATED TRANSPARENCIES.

EUT ReYOrt Bi-E-i72. 1987. ISBN 90-6144-172-2

(:Ti) Zhis $\mathrm{Yu}-\mathrm{Cad}$

ON A EOUND OF THE MODELIING ERRORS OE BLACK-BOX TRANSFER FUNCTION FSTIMATES. EUT Report 87-E-173. 1967. ISBN $90-5144-173-0$ 